\title{
Laser Polishing of Laser Powder Bed Fusion AlSi10Mg Parts-Influence of Initial Surface Roughness on Achievable Surface Quality
}

\author{
Markus Hofele1,2, Andre Roth', Jochen Schanz',2, David K. Harrison², Anjali K. M. De Silva², \\ Harald Riegel1
}

${ }^{1}$ Aalen University, Aalen, Germany

${ }^{2}$ Glasgow Caledonian University, Glasgow, UK

Email: markus.hofele@hs-aalen.de

How to cite this paper: Hofele, M., Roth, A., Schanz, J., Harrison, D.K., De Silva, A.K.M. and Riegel, H. (2021) Laser Polishing of Laser Powder Bed Fusion AlSi10Mg Parts-Influence of Initial Surface Roughness on Achievable Surface Quality. Materials Sciences and Applications, 12, 15-41. https://doi.org/10.4236/msa.2021.121002

Received: November 30, 2020

Accepted: January 17, 2021

Published: January 20, 2021

Copyright $\odot 2021$ by author(s) and Scientific Research Publishing Inc. This work is licensed under the Creative Commons Attribution International License (CC BY 4.0).

http://creativecommons.org/licenses/by/4.0/

\section{(c) (i) Open Access}

\begin{abstract}
Laser Powder Bed Fusion (LPBF) is an Additive Manufacturing technique, which allows production of highly complex solid metal parts with good mechanical properties, compared to conventionally manufactured parts. Nevertheless, the layer-by-layer fabrication process also offers several disadvantages, including a relatively high surface roughness depending on the shape of the component, its position and orientation during the fabrication process. This paper deals with investigations on the surface roughness reduction capability, and residual surface structures by laser polishing of LPBF AlSi10Mg parts under varying initial surface roughness in order to investigate the influence of the surface behavior and initial surface roughness to the achievable surface quality by laser polishing. Hereto test specimens with varying fabrication orientations regarding to the built platform are printed and further polished. Thereby the initial arithmetic roughness varies between $19.2 \mu \mathrm{m}$ and $8.0 \mu \mathrm{m}$. It could be shown that the achievable surface roughness by laser polishing with continuous and pulsed laser radiation is increasing with rising initial roughness, but the relative roughness reduction is almost constant in the range of $95 \%-97.5 \%$. The analyzation of the residual roughness structures shows, that the main roughness differences is found in the middle and long structure wavelength regime, which are directly depending on the initial surface structures of $3 \mathrm{D}$ printing.
\end{abstract}

\section{Keywords}

Additive Manufacturing, Surface Roughness, Aluminium AlSi10Mg, Laser Polishing, Fabrication Orientation 


\section{Introduction}

The powder-bed based selective laser melting (SLM) process is still in an ongoing investigation and development. The production of individual and complex parts with high mechanical properties and a wide range of applicable metals are essential advantages of the SLM-technology [1] [2]. This technology permits the production of parts directly from a CAD model. Due to a greater geometric freedom, more complex part geometries can be realized compared to other manufacturing processes [3] [4]. Because of the layer-by-layer process and the complex geometrical shapes, however, high surface roughness and contaminated surfaces result [5], which is undesirable for most applications, especially in the medical sector, the food industry or in clean rooms. The remaining surface roughness depends on the shape of the part, the orientation and positioning during the fabrication process [3] [4] [6] [7]. In [3] a varying surface roughness for AlSi10Mg samples with a horizontal fabrication $(\mathrm{Ra}=6.88 \mu \mathrm{m})$, a fabrication angle of 45 degrees $(\mathrm{Ra}=8.38 \mu \mathrm{m})$ and 90 degrees $(\mathrm{Ra}=6.15 \mu \mathrm{m})$ were found. In [7] a difference in surface roughness was observed between vertical fabrication direction $(\mathrm{Ra}=16 \mu \mathrm{m})$ and horizontal fabrication direction $(\mathrm{Ra}=20 \mu \mathrm{m})$. Furthermore, the surface roughness depends on the energy density which is imposed by the Laser on the surface. By optimizing the linear energy density the surface roughness of vertical planes can be reduced by more than $70 \%$ [8].

Several options such as laser parameters [9], laser beam oscillation strategies [10], laser remelting of each layer [11] and hatching strategies [12] have been investigated to reduce the surface roughness resulting from the SLM process. Nevertheless, the mentioned strategies and optimization possibilities improve the achievable surface qualities in the SLM process not sufficiently for most industrial applications.

Post-processing of additive manufactured metal components is typically done by machining with geometrically defined or undefined cutting edges (e.g. turning and milling or manual grinding and polishing). New alternative post-processing methods such as electron beam polishing [13], laser shock processing [14] [15], liquid jet polishing [16], abrasive blasting [17], laser ablation [18], mechanical polishing [19] or chemical polishing [20] have been considered. The post-processing methods mentioned have several disadvantages like environmental constraints, non-contact free and non-selective processing, loss of accuracy due to geometric restrictions caused by the post-processing technology and wastage of expensive material in common.

As a productive post-processing method for the reduction of the surface roughness and structure of the SLM process, laser polishing [21] has proven. It is contactless and exhibits a comparable degree of geometric freedom as the selective laser melting process itself [22]. The polishing process uses a laser beam to melt a thin surface layer of the part to be processed (Figure 1).

Due to the surface tension and capillary forces of the liquid material, the surface structure is smoothened by a material flow from peaks to valleys [23]. 


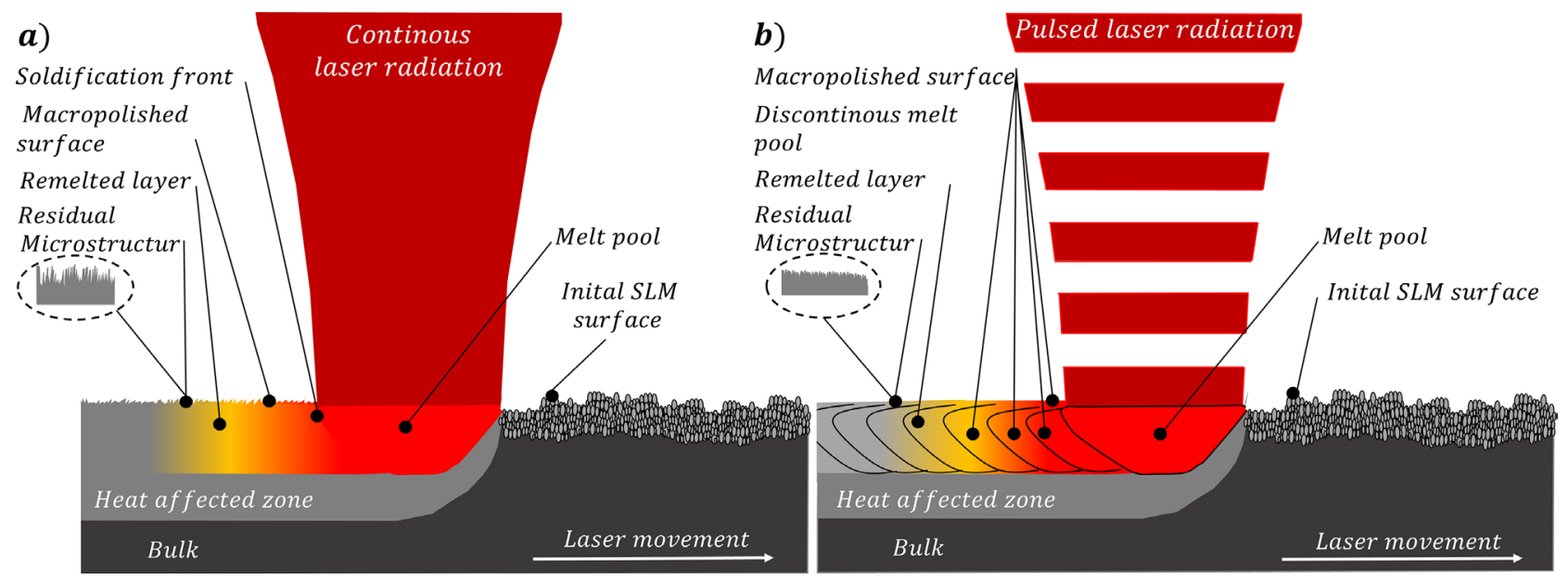

Figure 1. Simplified scheme of laser remelting with (a) continuous laser radiation and (b) pulsed laser radiation.

Underneath the remelted layer a heat-affected zone is generated. Common modes of laser operation are continuous wave (Figure 1(a)) and pulsed wave (Figure 1(b)). The two modes of operation differ mainly in the solidification point of the melt pool. In the pulsed operating mode, the previously melted material solidifies partially or completely while in the continuous operating mode the cooling and solidification front of the material is constantly following the laser spot. Concerning the roughness spectrum laser polishing is generally divided into micro and macro polishing [13] [23]. For long roughness wavelength structures, macro-polishing is applicable, which is commonly performed with continuous laser radiation (Figure 1(a)), a treatment by pulsed laser radiation with pulse durations in the millisecond range is also applicable (Figure 1(b)) [23] [24]. For improvement of the gloss level and the roughness of short spatial wavelengths under $80 \mu \mathrm{m}$, micro polishing can be used, which is typically carried out with short or ultrashort laser pulses with a pulse duration in the range of several $\mu$ s down to ps [13] [25].

Laser polishing of additively manufactured flat parts is investigated on stainless steel 316L [4] [26], Cobalt-Chromium alloys (CoCr) [27] [28] [29], ASTM F75 [30], Inconel IN 718 [30] [31] [32], ST-100 [33] [34], AlSi tool steel H13 [35], Titanium Ti6Al-4V [36] [37], Aluminium AlSi10Mg [38] [39], AlSi420 stainless steel [33], Ti-6.5Al-3.5Mo-1.5Zr-0.3Si (TC11) [36] and SLM Fe-Ni steel [40]. For AlSi10Mg flat test specimens a roughness of $\mathrm{Ra}=0.66 \mu \mathrm{m}$ was achieved in [39]. For Ti6Al-4V a roughness of down to $\mathrm{Ra}=0.32 \mu \mathrm{m}$ was achieved in [37]. Depending on investigated materials, laser parameters and process strategies, relative roughness reductions of $50 \%-90 \%$ were achieved, assuming an initial and untreated surface roughness of approximately. 8 to $22 \mu \mathrm{m}$ in the mentioned investigations.

To sum up, surface polishing of commonly used materials for LPBF were mostly done on flat specimens and significant surface improvements are shown. Industrial 3D parts typically exhibit complex freeform surface geometries. The orientation of those parts at the LPBF in relation to the built platform, steadily 
varying stair-effects of the layer wise production and overhanging geometries occur, which leads to strongly inhomogeneous surface behavior. The systematic analyzation of these varying SLM surfaces on the roughness reduction capability, the process efficiency and residual surface structures is still an open field for investigation.

This paper deals with investigations of the influence of the surface behavior and initial surface roughness of AlSi10Mg parts, made by laser powder bed fusion (LPBF), on the achievable surface quality by laser polishing. Hereto flat samples are produced under varying fabrication angle, regarding to the base plate at the LPBF process. The initial surface topography of the 3D printed parts and the Laser polished surfaces are qualitatively and quantitatively analyzed by means of 3D profilometer and tactile roughness measurements. Laser polishing is done by continuous and pulsed laser radiation in order to compare the process stability at varying initial surface behavior and the roughness reduction capability between the laser operation modes.

\section{Experimental Setup}

\subsection{Material and Samples}

In this paper experimental investigations are carried out with the aluminum alloy AlSi10Mg. To investigate the influence of the initial surface structure on the polishing result, rectangular plates with a length of $100 \mathrm{~mm}$, a width of $30 \mathrm{~mm}$ and a material thickness of $3 \mathrm{~mm}$ were built up through Selective Laser Melting (SLM) on a SL280HL SLM-machine (SLM Solutions GmbH) as test specimens. The SL280HL is equipped with a $400 \mathrm{~W}$ Yb fiber laser and a fabrication chamber with the dimensions $280 \times 280 \times 280 \mathrm{~mm}^{3}$. The average powder grain diameter of the aluminum powder used is $37 \mu \mathrm{m}$. The fabrication of the samples was carried out with a slicing thickness of $50 \mu \mathrm{m}$ and recommended building parameters, which were provided by SLM Solutions GmbH. With a laser power of 350 $\mathrm{W}$ and a beam velocity of $600 \mathrm{~mm} / \mathrm{s}$ the layers were melted on the outer contour. For the core of the part a laser power of $350 \mathrm{~W}$, a beam velocity of $930 \mathrm{~mm} / \mathrm{s}$ and a hatch distance of $170 \mu \mathrm{m}$ was used.

The samples were orientated in different angles form horizontally to vertically by steps of $\Delta \alpha=15^{\circ}$ degrees. Due to the layer wise fabrication of the samples, the resulting surface topography and roughness is variating strongly [3] [6]. Parts with an orientation angle (fabrication angle) of $15^{\circ}$ and $30^{\circ}$ degrees were built up with a support structure underneath. For each orientation angle, four samples were built on the base plate with varying positions (Figure 2).

In order to remove most of the oxide layers resulting from the fabrication process as well as the residual powder particles within the rough initial surfaces without ablation or modification of the bulk material, the samples were laser cleaned before laser polished. This was performed with a TruMark 5020 short-pulse laser with a scanning speed of $3000 \mathrm{~mm} / \mathrm{s}$, a pulse duration of $70 \mathrm{~ns}$ and a pulse frequency of $65.5 \mathrm{kHz}$. 


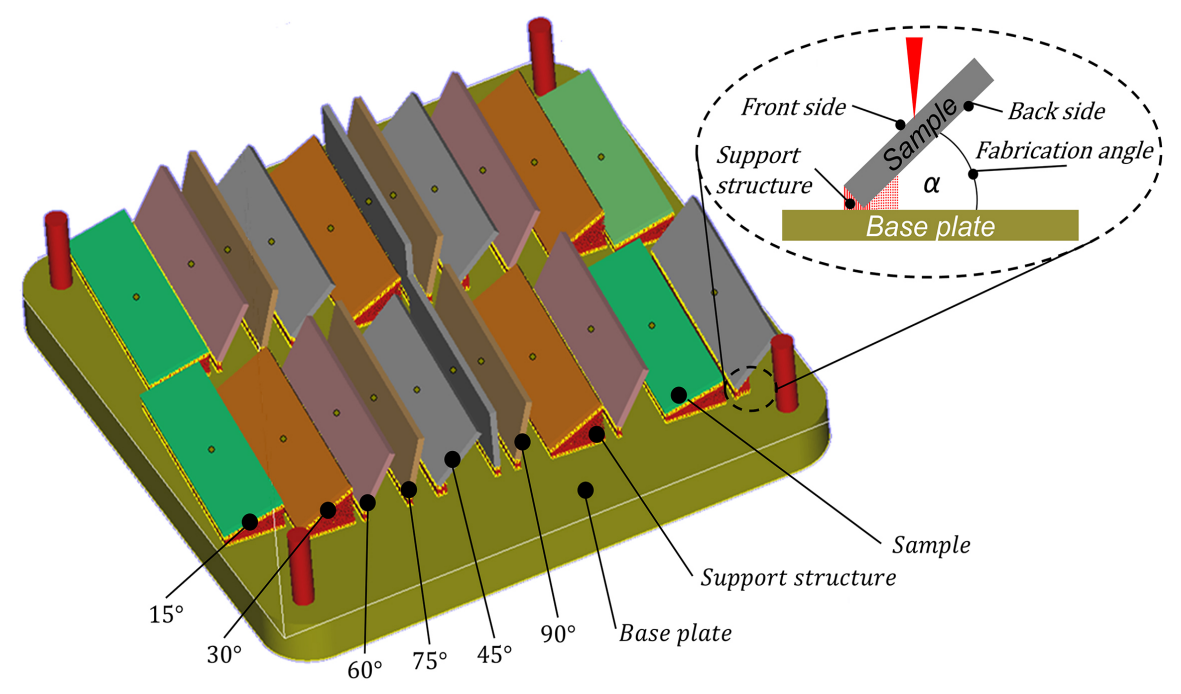

Figure 2. Position and orientation of the Aluminium samples on the SLM-building platform (Base plate).

\subsection{Polishing Setup and Process Parameters}

The experimental setup is shown in Figure 3. A TRUMPF disk laser of the type TruDisk4002 with a maximum output power of $4000 \mathrm{~W}$ was used. A $200 \mu \mathrm{m}$ gradient index fiber with a numerical aperture of $\mathrm{NA}=0.1$ was used to guide the laser beam to a modified 5-axis TRUMPF laser cell TLC40.

To protect the samples from atmospheric oxygen the work pieces are processed in a sealed process chamber under a purified inert gas atmosphere. The residual oxygen concentration was monitored during the laser polishing process with an Orbitalservice oxygen measuring device of the type $\mathrm{PRO} 2$ plus.

The beam is guided by a scanner optic of the type SAO 1.06/1D (Figure 3). The pendulum movement is realized by a $1 \mathrm{D}$ scanner system, which enables a maximum pendulum length of $70 \mathrm{~mm}$ at frequencies between $10-300 \mathrm{~Hz}$. Figure 4 shows the schematic pendulum movement of the laser beam over the surface via scanner optics in conjunction with the machine axis.

Thereby the pendulum movement in $y$-direction is superimposed by the axis federate of the scanner head in $\mathrm{x}$-direction. At the two turning points, resulting from the $1 \mathrm{D}$ pendulum movement, the energy input into the material would be significantly increased at constant laser power. This would increase the melting depth and process instabilities could occur. This was avoided by dividing the pendulum axis into 15 segments with adjustable laser power, see Figure 5. Preliminary investigations have shown that a reduction of the laser power by $200 \mathrm{~W}$ and finally $400 \mathrm{~W}$ in both turning points proves to be effective, resulting in an almost constant melt pool depth.

At laser polishing the plates were mounted horizontally on a clamping plate (see Figure 4). The x-direction is equal to the sample vertical direction (SVD). Thus the laser beam is steadily vertically orientated regarding to the sample surface in $\mathrm{z}$-direction over all sample, respectively over all fabrication angles. 
The basic laser process parameters of the applied laser operation modes (pulsed and continuous), used in this study, are based on previous investigations [38] [41]. The polishing was done on quadratic test fields with a dimension of 10 $\times 10 \mathrm{~mm}^{2}$. General parameters and specific process parameters for continuous and pulsed laser polishing within this study are shown in Table 1.

The used laser power as a function of pendulum axis segments is shown in Figure 5.

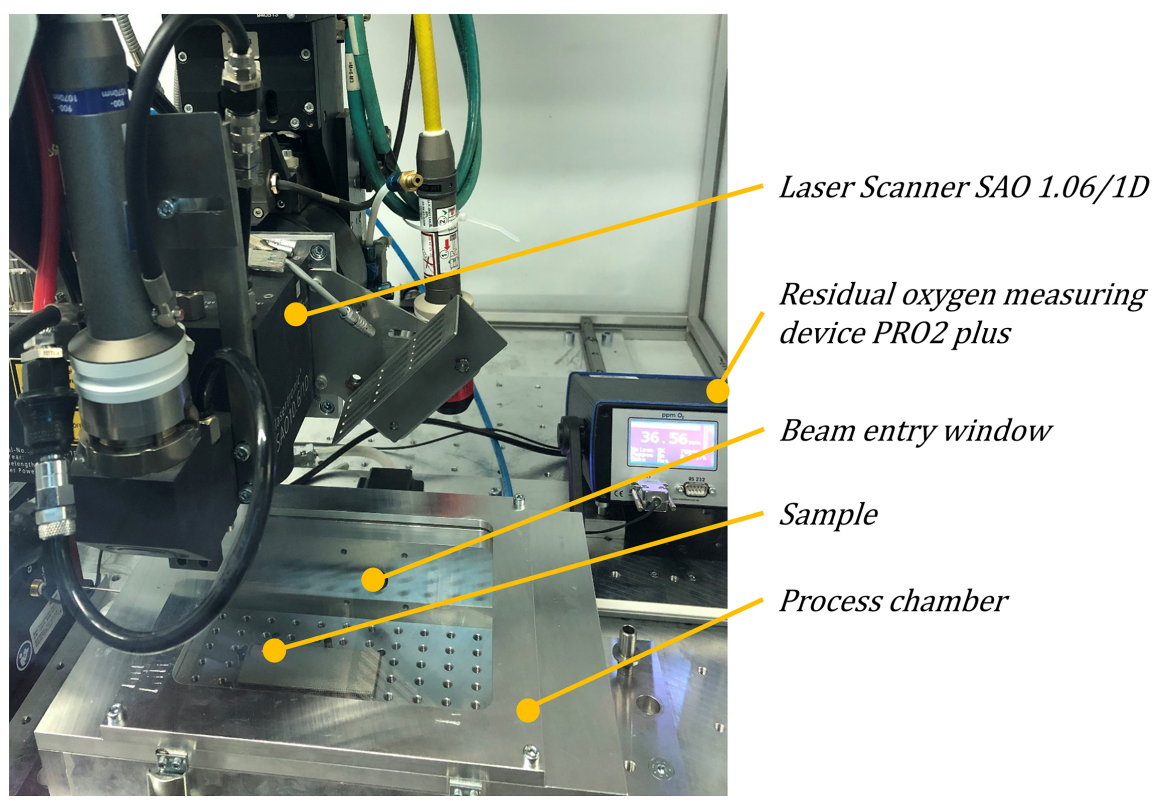

Figure 3. Experimental setup, Trumpf Laser Cell TLC 40, process chamber, oxygen measurement device PRO2 plus and SAO 1.06/1D scanner optic.

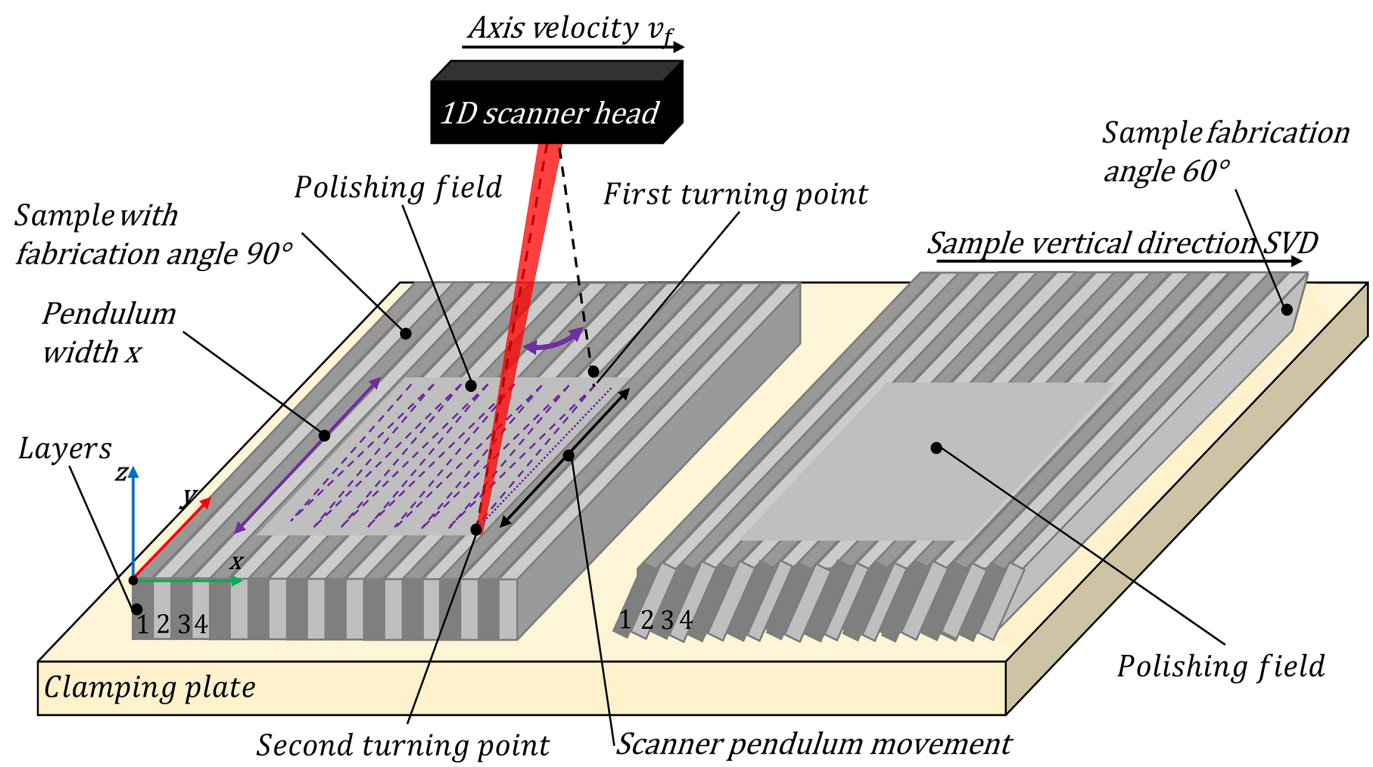

Figure 4. Left: Schematic description of the beam guidance by means of a 1D scanner and superimposed axix movement. Sample orientation on the clamping plate in the process chamber during laser polishing, left: $90^{\circ}$ degree fabrication angle, right: $60^{\circ}$ degree fabrication angle. 
Table 1. General parameters and specific process parameters for laser polishing.

\begin{tabular}{|c|c|c|c|}
\hline & & \multicolumn{2}{|c|}{ Laser Operation Mode } \\
\hline & & Pulsed & Continuous \\
\hline \multirow{4}{*}{$\begin{array}{c}\text { General } \\
\text { Parameters }\end{array}$} & Pendulum width $[\mathrm{mm}]$ & \multicolumn{2}{|c|}{10} \\
\hline & Process gas flow rate $[1 / \mathrm{min}]$ & \multicolumn{2}{|c|}{15} \\
\hline & Residual Oxygen [ppm] & \multicolumn{2}{|c|}{40} \\
\hline & Process gas & \multicolumn{2}{|c|}{ Argon } \\
\hline \multirow{11}{*}{$\begin{array}{c}\text { Specific Process } \\
\text { Parameters }\end{array}$} & Laser Power $[\mathrm{W}]$ & 1700 & 1400 \\
\hline & Pulse duration $[\mathrm{ms}]$ & 0.3 & - \\
\hline & Pulse frequency $[\mathrm{Hz}]$ & 1000 & - \\
\hline & average Power [W] & 510 & - \\
\hline & Beam intensity $\left[\mathrm{W} / \mathrm{mm}^{2}\right]$ & 1940 & 1600 \\
\hline & Energy density $\left[\mathrm{J} / \mathrm{mm}^{2}\right]$ & 76.5 & 33.6 \\
\hline & Avg. pulse overlap [\%] & 81.1 & - \\
\hline & Track overlap [\%] & 93.7 & $92.1 / 89.0$ \\
\hline & Focal position $[\mathrm{mm}]$ & \multicolumn{2}{|c|}{12} \\
\hline & Axis velocity $[\mathrm{mm} / \mathrm{min}]$ & 40 & $250 / 350$ \\
\hline & Pendulum frequency $[\mathrm{Hz}]$ & 10 & 50 \\
\hline
\end{tabular}

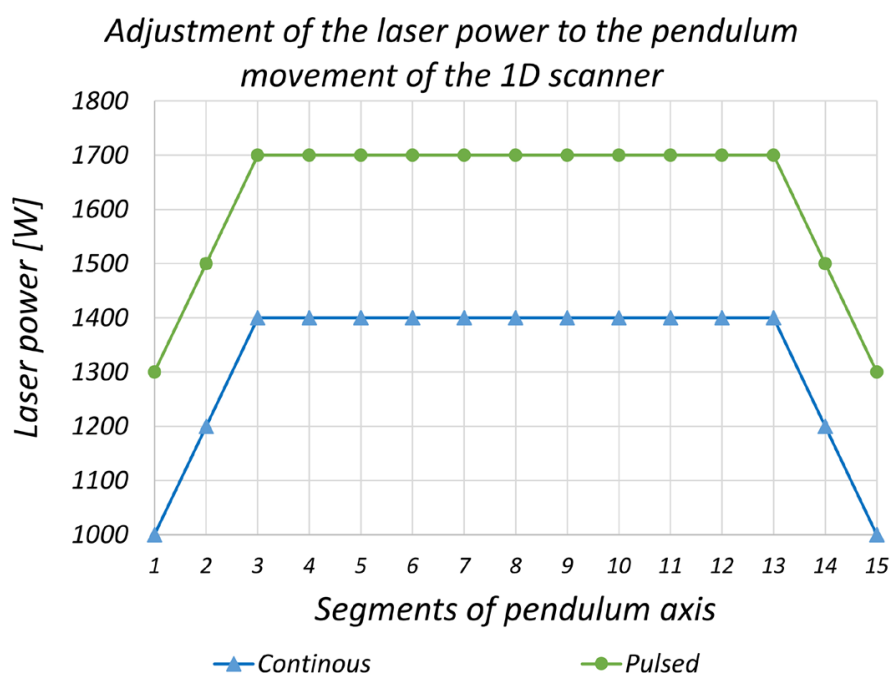

Figure 5. Laser power ramping during the laser polishing processes.

\subsection{Measuring Devices and Evaluation Methods}

The surfaces of the initial as well as the laser-polished surfaces were quantitatively and qualitatively investigated. The analysis of the quantitative surface roughness, given by the arithmetic roughness $\mathrm{Ra}$, was performed tactile according to EN ISO 4288:1997 using a MarSurf M400 perthometer. For initial surfaces with $\mathrm{Ra}>2 \mu \mathrm{m}$ a cut-off wavelength of $2500 \mu \mathrm{m}$ was used according to the ISO 4288 standard, while a cut-off wavelength of $800 \mu \mathrm{m}$ was used for laser-polished sur- 
faces with $\mathrm{Ra}<2 \mu \mathrm{m}$. Five measurements were taken for each polishing field and ten measurements for each of the initial surfaces, from which an average value was derived. Therefore, the positions of the individual measurements were distributed homogeneously over the polishing field respectively the test plates.

For a more detailed investigation of the surface structure, the spatial roughness is analyzed with a Fourier transformation. Hereto the tactile measured surface profile is leveled to zero and the tilt of the measurement is eliminated. After the Fourier transformation the roughness values are clustered into a logarithmic scale.

The surface topography and roughness of the initial and the polished surface were measured in sample vertical direction (SVD) of the SLM process (Figure 6). Thus, the dominating roughness structure of the layers have been considered. The laser polishing process is carried out by an axis movement perpendicular to the sample vertical direction and a scanner beam deflection in sample vertical direction (Figure 6).

The qualitative evaluations of the surface topographies were performed by a 3D profilometer VR-3100 (Keyence). This measurement was used to perform an optical evaluation of the 3D surface topography. The surface appearance and the microstructures are analyzed by means of a microscope of type Axio Zoom V16 (Zeiss).

\section{Results and Discussion}

\subsection{Initial Surface Characterization}

After cutting the samples from the 3D printing base plate, the SLM surfaces roughness were measured in sample vertical direction (SVD). Figure 7 shows the dependency on the sample angle and differences between the frontside and the overhanging backside surface on the surface roughness Ra.

\section{Frontview}

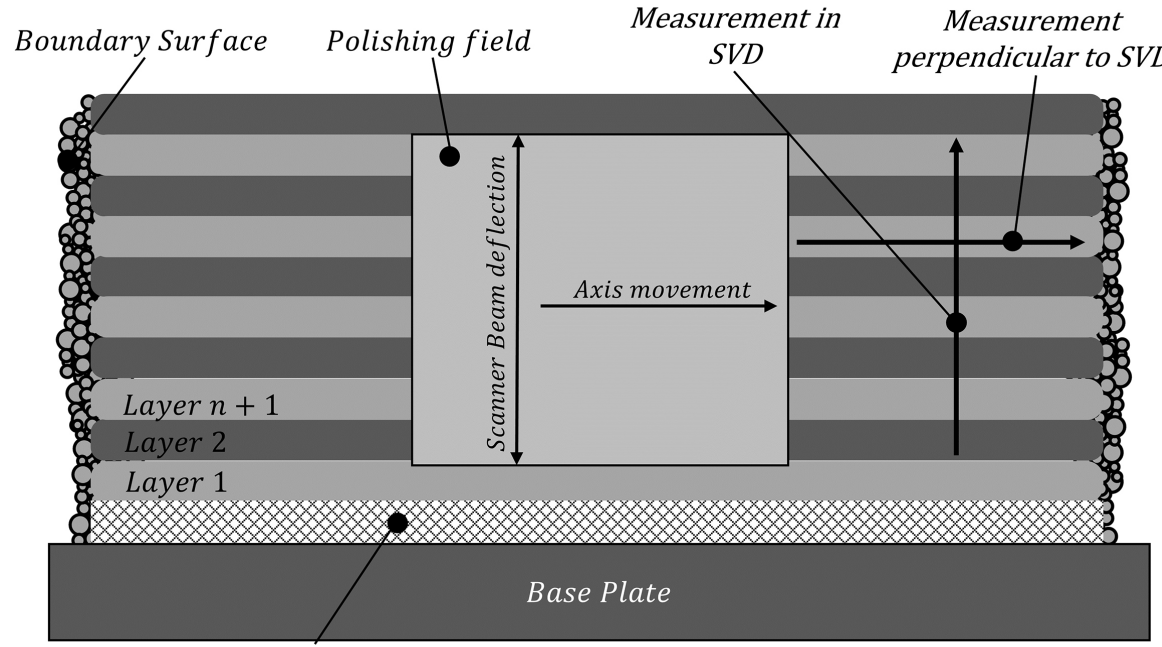

Support structure
Sideview

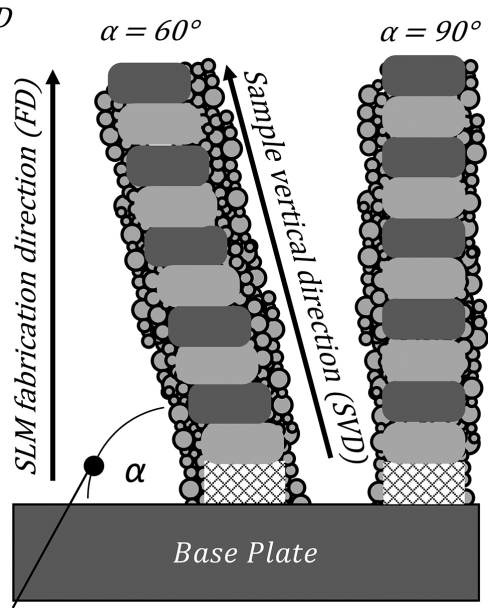

Fabrication angle

Figure 6. Definition of the LPBF fabrication, polishing and measurement directions on the AM samples. 


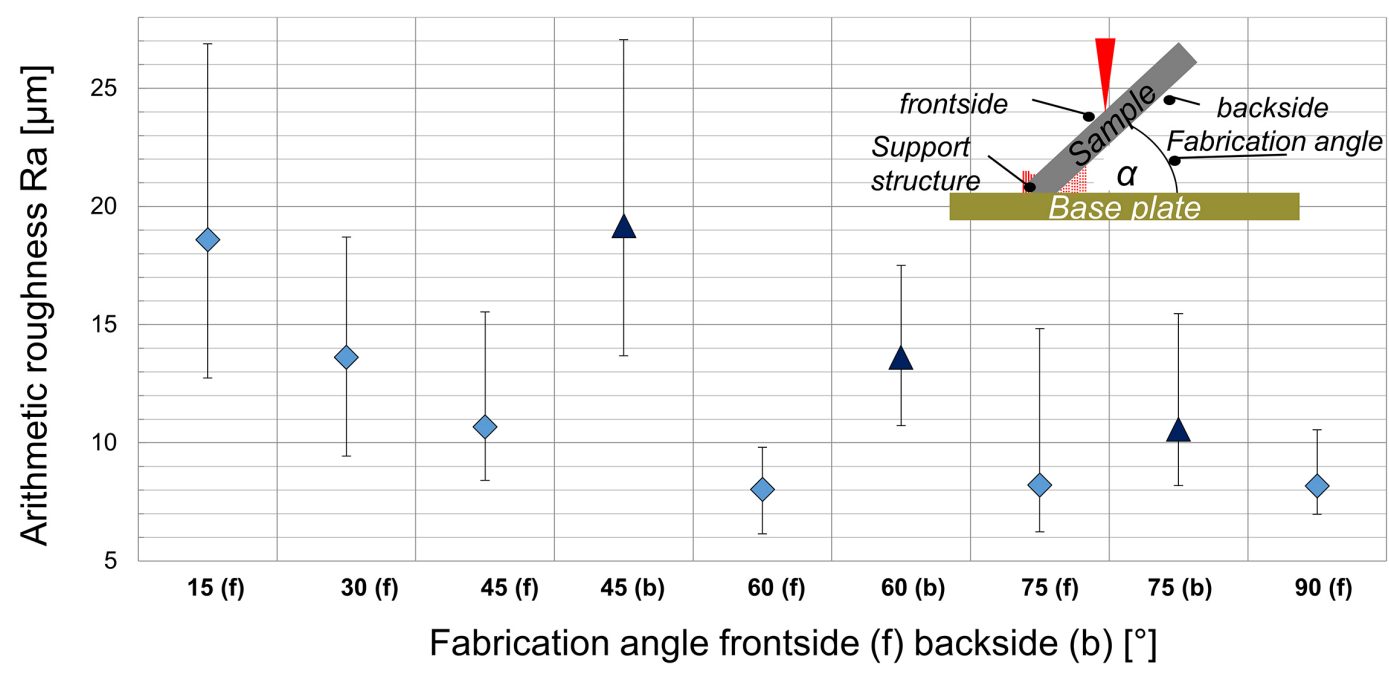

Figure 7. Arithmetic roughness Ra of intial samples according to DIN EN ISO 4288 depending on the fabrication angle and the side of the specimens.

The roughness $\mathrm{Ra}$ of the frontside of the samples is decreasing from $\mathrm{Ra}=18$ $\mu \mathrm{m}$ at 15 degrees to $\mathrm{Ra}=7 \mu \mathrm{m}$ at 60 degrees. Further increasing of the fabrication angle has no significant influence. At $45^{\circ}$ degree the arithmetic roughness on the overhanging backside of the sample is with $\mathrm{Ra}=19 \mu \mathrm{m}$ double as high compared to the front side of the part. The roughness variation, shown by the scattering bars, is at arithmetic roughness Ra (Figure 8) above $15 \mu \mathrm{m}$ bigger than below $15 \mu \mathrm{m}$. Similar dependencies between the resulting surface roughness and the fabrication orientation at LPBF where found on AlSi10Mg [3] [7].

A qualitative analysis of the initial roughness, by $3 \mathrm{D}$ topography measurement, gives an impression of what causes the main differences of the surface roughness (Figure 8). It should be noted that the height scale differs between the images. At $15^{\circ}$ degrees, in the upper left picture, the stairs effect of the layer wise fabrication technique is clearly visible. The linear parallel running welding beads superimposed by spatter induced material accumulations are causing the main surface roughness structures with maximum variation of height up to $170 \mu \mathrm{m}$. With increasing fabrication angle, the stairs effect is no more visible and the surface is getting comparable flat. At $90^{\circ}$ degrees, the in-homogeneously distributed melt pool accumulations cause the dominant roughness components and the maximum variation of the surface height deviation gets decreased. The surface on the overhanging 45 degree backside shows massive densely packed material accumulations with expansions of several hundred $\mu \mathrm{m}$, which are responsible for the highest measured arithmetic roughness $\mathrm{Ra}$ of those test batches.

A deeper analyzation of the varying roughness profiles depending on the sample vertical directions (SVD) is given by the analysis of the Fourier transformation. The following diagram (Figure 9) shows the partial roughness Ra depending on the structural wavelength on double logarithmic scales. Over all sample vertical directions (SVD), the main roughness amounts are present in the long structure wavelength of greater than $150 \mu \mathrm{m}$. At short structural wavelength below $40 \mu \mathrm{m}$ 


\section{a)}

$3 \mathrm{D}$ topography sample angle $15^{\circ}$ (f)

$\mathrm{Ra}=18.6 \mu \mathrm{m}$

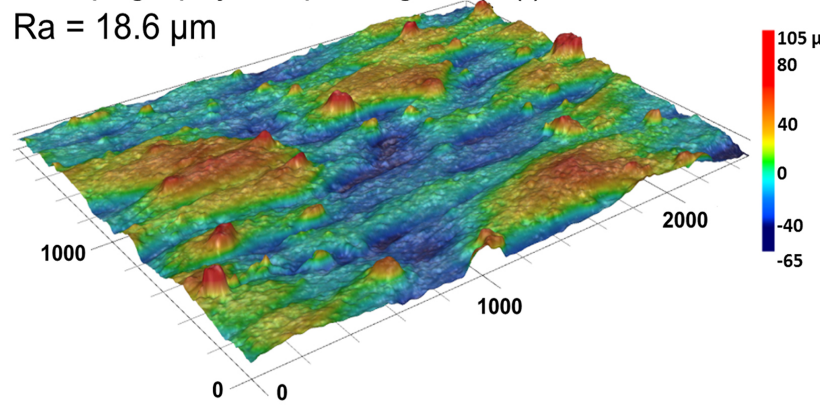

b)

$3 \mathrm{D}$ topography sample angle $90^{\circ}$ (f)

$\mathrm{Ra}=11.2 \mu \mathrm{m}$

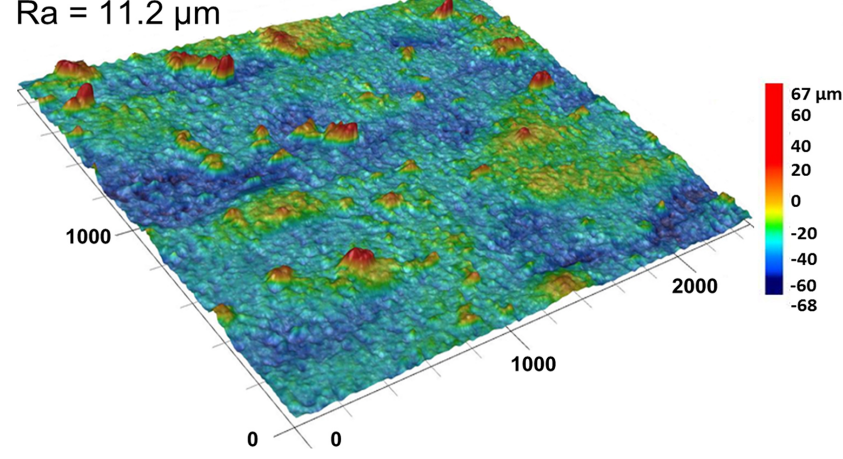

c)

3D topography sample angle $45^{\circ}(\mathrm{f})$

$\mathrm{Ra}=10.6 \mu \mathrm{m}$

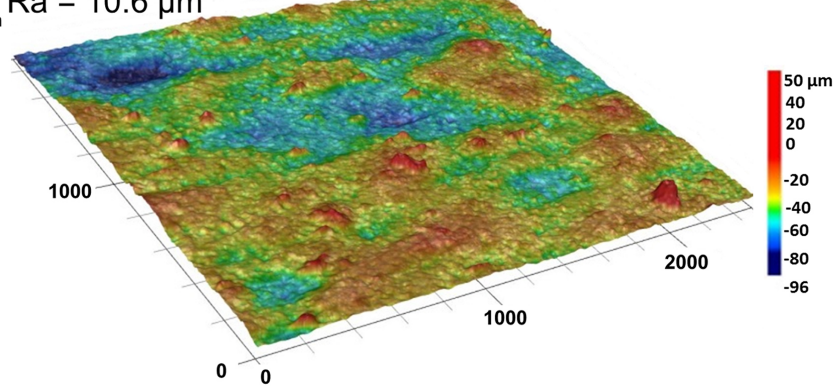

d)

3D topography sample angle $45^{\circ}$ (b)

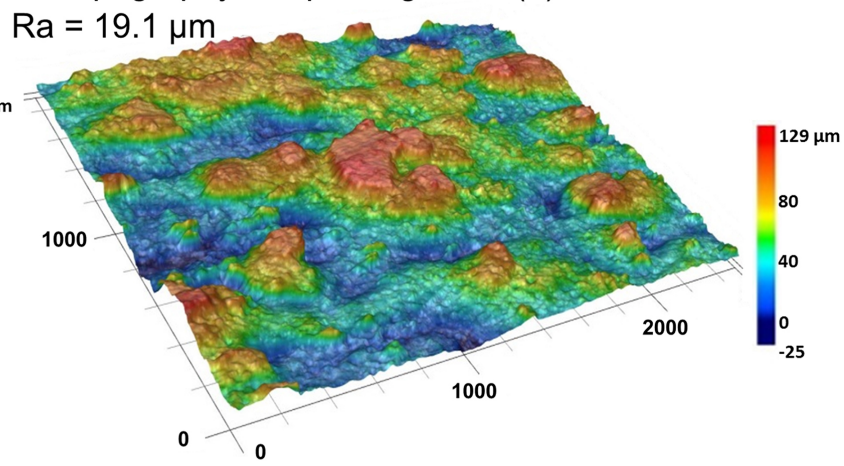

Figure 8. Qualitative comparison of the initial surface topography (a) Fabrication angle $15^{\circ}$ frontside (f), (b) Fabrication angle $90^{\circ}$, (c) Fabrication angle $45^{\circ}$ frontside (f), (d) Fabrication angle $45^{\circ}$ backside (b). It should be noted that the height scale differs between the images.

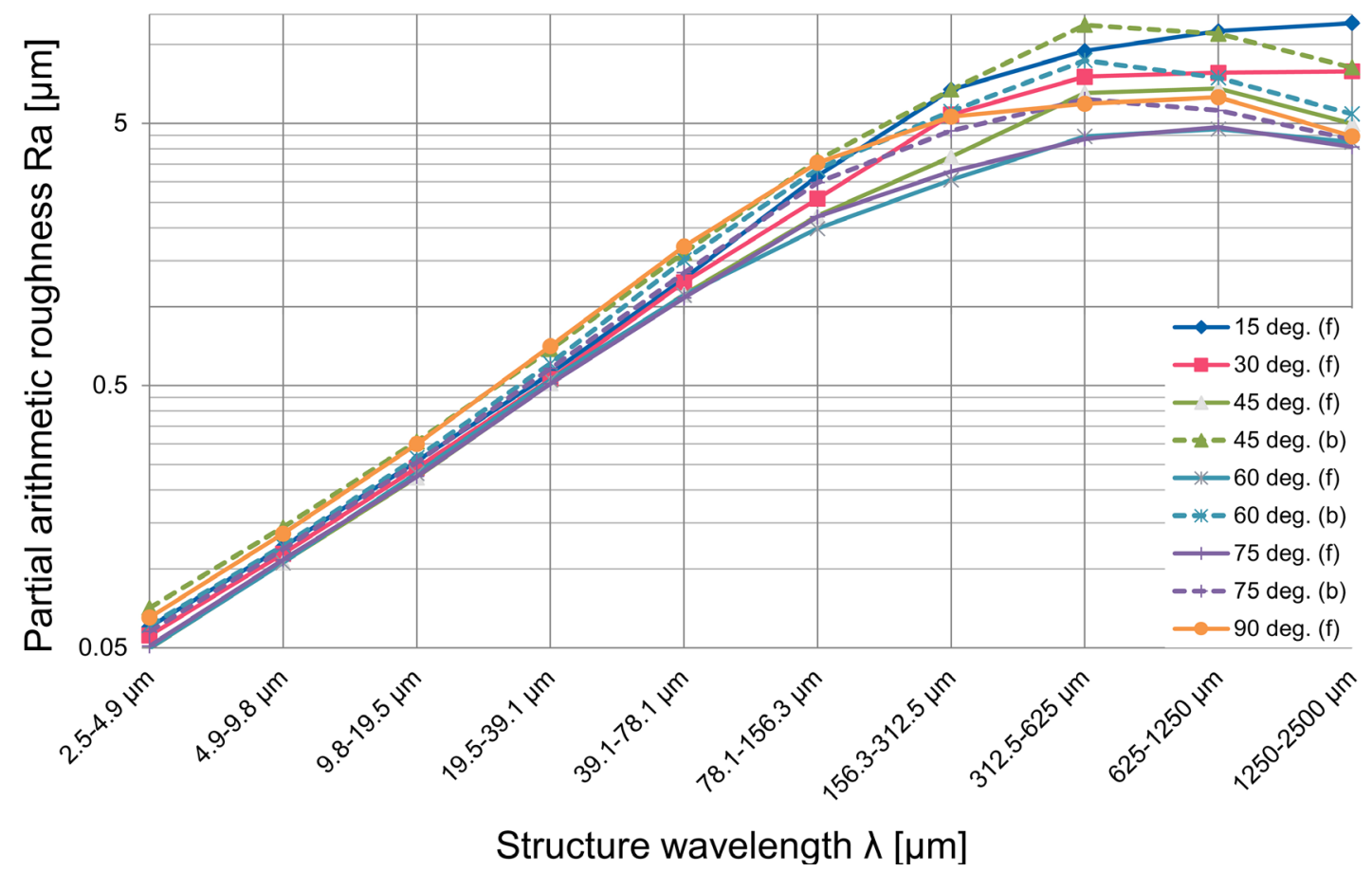

Figure 9. Initial surface roughness over structure wavelength by varying fabrication angle at frontside (f) and backside (b), measured in sample vertical direction (SVD). 
the fabrication angle has a comparatively small influence on the existing roughness. With increasing structural wavelength the partial surface roughness is significantly increased with small fabrication angles $\left(15^{\circ}\right.$ and $\left.30^{\circ}\right)$ and overhanging surfaces (backside-b).

\subsection{Pulsed Mode Laser Polishing}

In order to analyze the influence of the fabrication angle and the initial surface structures after additive manufacturing, pulsed mode laser polishing is done with constant process parameters, mentioned in Table 1 with one and two polishing passes. The roughness Ra for laser polishing with one and two crossings is given in Figure 10.

Figure 10 shows that the roughness of the laser polished samples is in a range between $1.0 \mu \mathrm{m}$ and $0.2 \mu \mathrm{m}$. It is reduced tendential at increasing fabrication angle. At a fabrication angle of $15^{\circ}$, a roughness of $\mathrm{Ra}=0.62 \mu \mathrm{m}$ was achieved with one crossing (yellow triangle, measured in SVD). By increasing the fabrication angle up to $75^{\circ}$ the roughness was reduced to a minimum of $\mathrm{Ra}=0.32 \mu \mathrm{m}$ (one crossings measured in SVD). In addition, the roughness measured in the sample vertical direction, respectively parallel to the fast scanner pendulum movement, is consistently (about $1 / 3$ ) lower than the roughness, measured perpendicular to SVD. The residual average surface roughness after laser polishing regarding to all fabrication angles is further reduced by a second crossing (average Ra in SVD $=0.33 \mu \mathrm{m}$; average Ra perpendicular to SVD $=0.43 \mu \mathrm{m}$ ), compared to one crossing (average Ra in SVD $=0.45 \mu \mathrm{m}$; average Ra perpendicular to SVD $=0.76$ $\mu \mathrm{m})$. The roughness improvement by a second polishing pass is significant

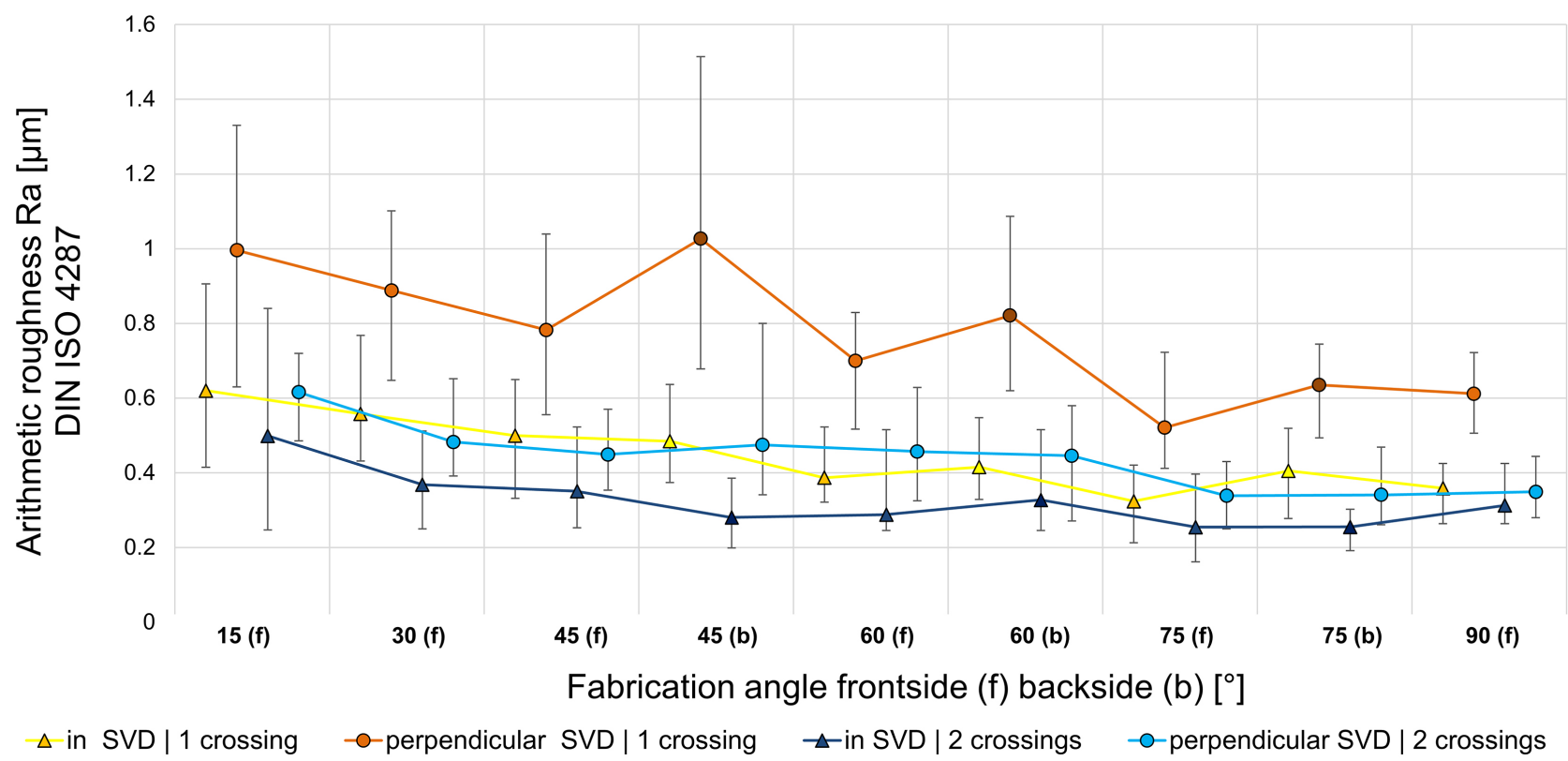

Figure 10. Arithmetic roughness Ra of laser polished samples with pulsed radiation according to DIN ISO 4287 measured in and perpendicular to the sample vertical direction (SVD) with respect to fabrication angle for one and two crossings with an axis velocity of $40 \mathrm{~mm} / \mathrm{min}$. 
greater perpendicular to the sample vertical direction. Hence the gap between the measurement directions are decreased and a more direction independent surface quality is achieved. The maximum deviation, visualized by the scatter bars, shows that the achievable roughness is strongly linked to the initial roughness, since the scatter range decreases with increasing fabrication angle, for the initial roughness as well as the achieved roughness.

The relative roughness reduction $\left(\mathrm{Ra}_{\text {initial }}-\mathrm{Ra}_{\text {laser polished }}\right) / \mathrm{Ra}_{\text {initial }}$ based on an initial roughness of $\mathrm{Ra}_{\text {initial }}$ according to Figure 7 and the results in Figure 10 is given in Figure 11.

Figure 11 shows that the roughness reduction, considered over all fabrication angles and measuring directions, is more or less independent of the fabrication angle. In addition, no significant tendency can be seen that the smoothing ability is strongly dependent on the initial roughness. It becomes clear from Figure 11 that a further roughness reduction, at both measuring directions, can be achieved by a second crossing over all fabrication angles. Moreover, a further roughness reduction measured perpendicular to SVD compared to the measurement in the SVD can be achieved by a second crossing. For the respective backsides of the samples over all construction angles a higher roughness reduction can be determined for both measuring directions compared to roughness reduction on the frontsides. For example, a relative roughness reduction of up to $98.5 \%$ (measured in SVD) can be achieved for a fabrication angle of $45^{\circ}$ (b) with two crossings.

The analyzation of the existing partial roughness over the structure wavelength after pulsed mode laser polishing with varying fabrication angle, measured in sample vertical direction, is given in Figure 12.

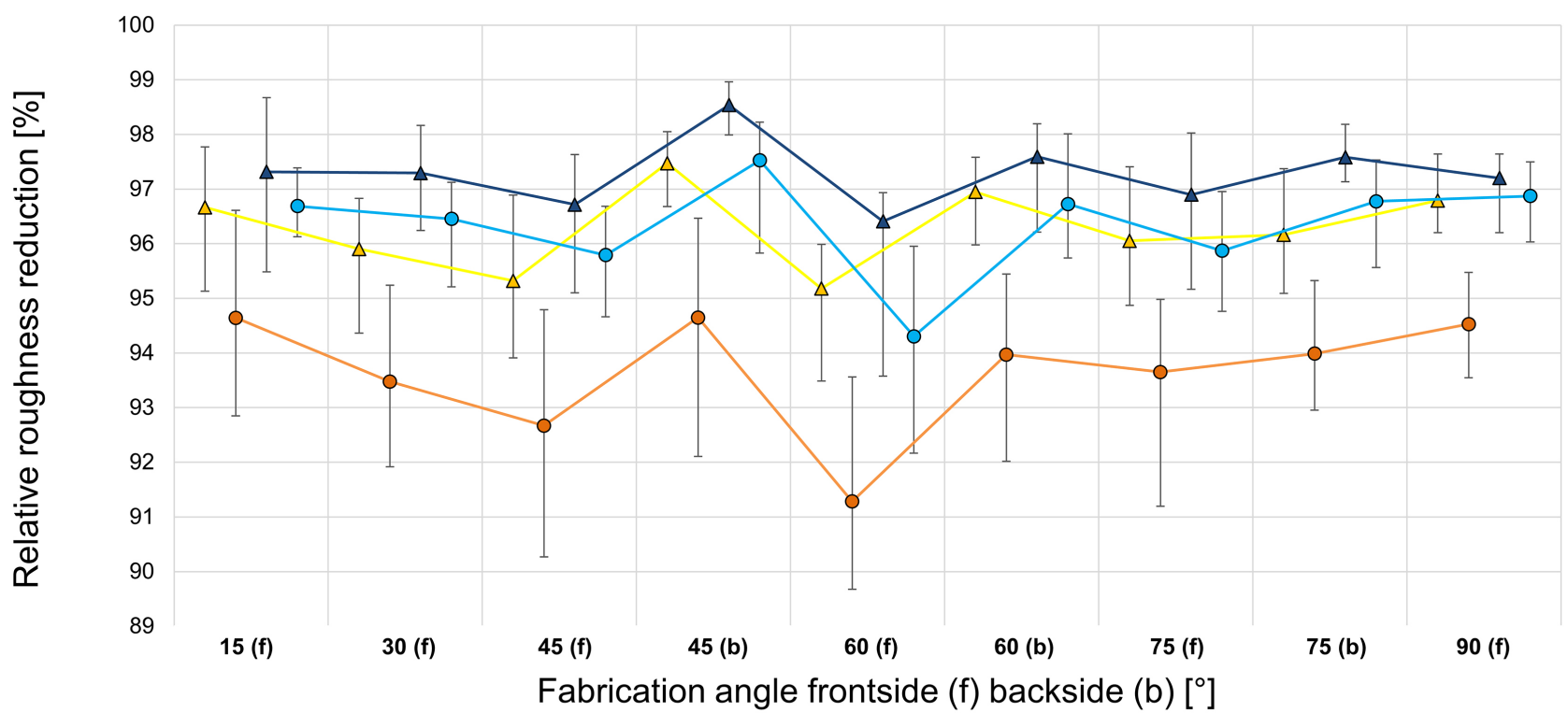

$\Delta$ in SVD $\mid 1$ crossing - - -perpendicular SVD $\mid 1$ crossing $\triangle-$ in SVD $\mid 2$ crossings - - -perpendicular SVD $\mid 2$ crossings

Figure 11. Roughness reduction in percent measured in and perpendicular to the sample vertical direction (SVD) for one and two crossings with pulsed radiation under varying the fabrication angle. 


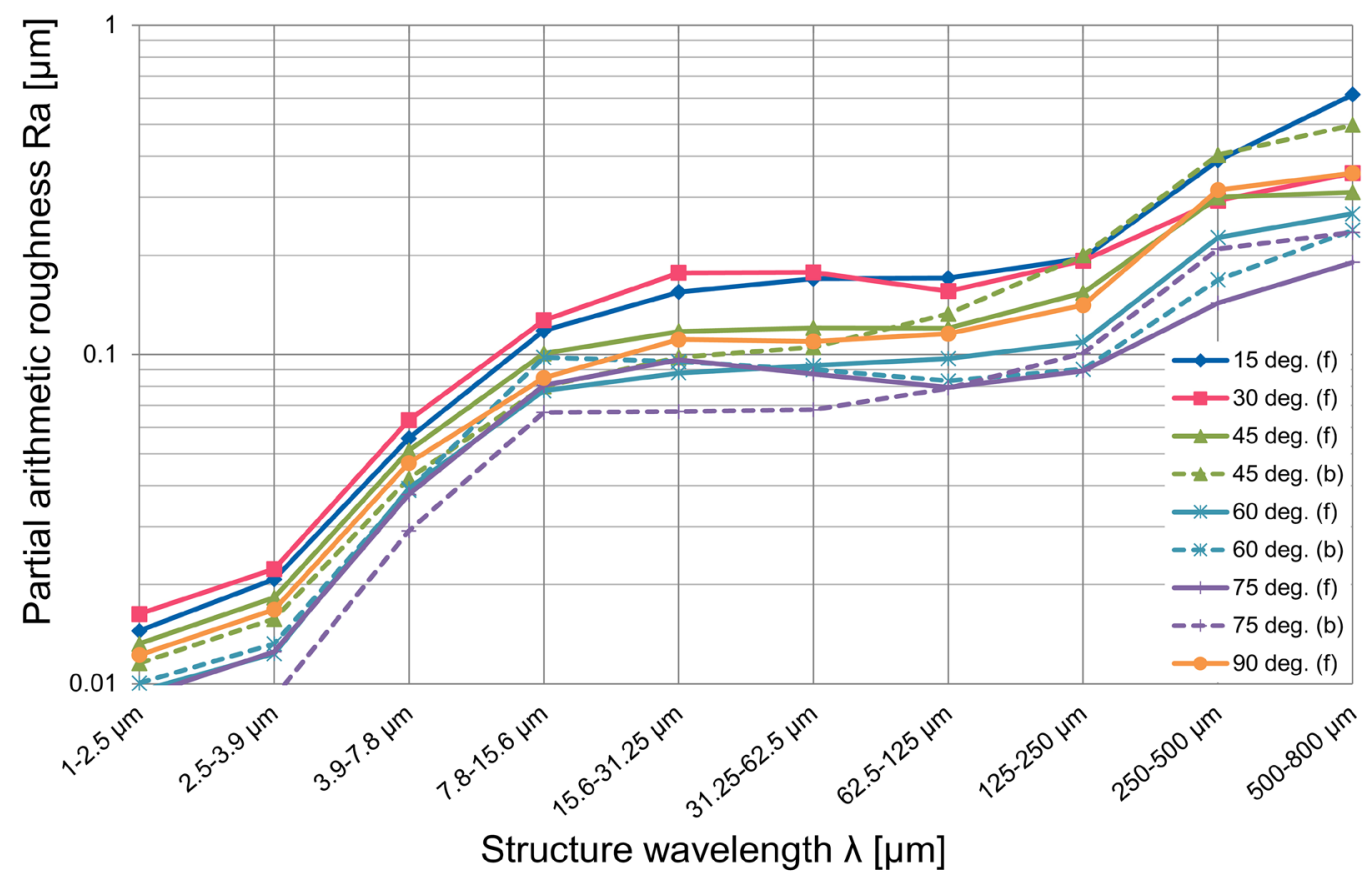

Figure 12. Partial roughness over structure wavelength after pulsed mode polishing: Influence of the fabrication angle frontside (f), backside (b) with two polishing passes, measured in SVD.

The residual partial surface roughness at the fabrication angles $15^{\circ}$ and $30^{\circ}$ degree is significantly increased in the medium structure wavelength range between 7.8 - $125 \mu \mathrm{m}$, compared to bigger fabrication angles. Hence, the roughness for a fabrication angle of $15^{\circ}$ (f) in the range of $15.6-31.25 \mu \mathrm{m}$ is $\mathrm{Ra}=0.17 \mu \mathrm{m}$ while in the same range the roughness for a fabrication angle of $60^{\circ}$ (f) is $\mathrm{Ra}=$ $0.09 \mu \mathrm{m}$. Also it can be seen, that the backside surface with $45^{\circ}$ degree can be improved much better, compared to smaller fabrication angles. At the long wavelength structures above $125 \mu \mathrm{m}$ the residual partial roughness correlates to the initial surface roughness. In this range, the achievable surface quality is directly depending on the initial roughness. Hence the achieved partial surface roughness varies between $0.19 \mu \mathrm{m}$ and $0.61 \mu \mathrm{m}$ at the structure wavelength range between $500-800 \mu \mathrm{m}$.

Differences in the surfaces appearance and surface structures between the different fabrication angles with one and two polishing passes can be taken from the microscopic images in Figure 13. Comparing the roughness, regarding the number of crossings, it becomes clear that the polishing with two crossings leads to a better recognizable roughness improvement at all sample vertical directions. While the vertical scanner hatches of the laser in pendulum direction (y-direction) are clearly visible (e.g. Figure 13(a) and Figure 13(e)) with one crossing, at two crossing those residual structures are smoothed (Figure 13(b) and Figure 13(f)). Also, it becomes visible that the surface is full of black areas which could indicate oxide layers, however an EDX-analysis was not performed. Furthermore, crusted surface structures were observed. With an increasing fabrication angle the size of the Oxide areas is decreasing. 


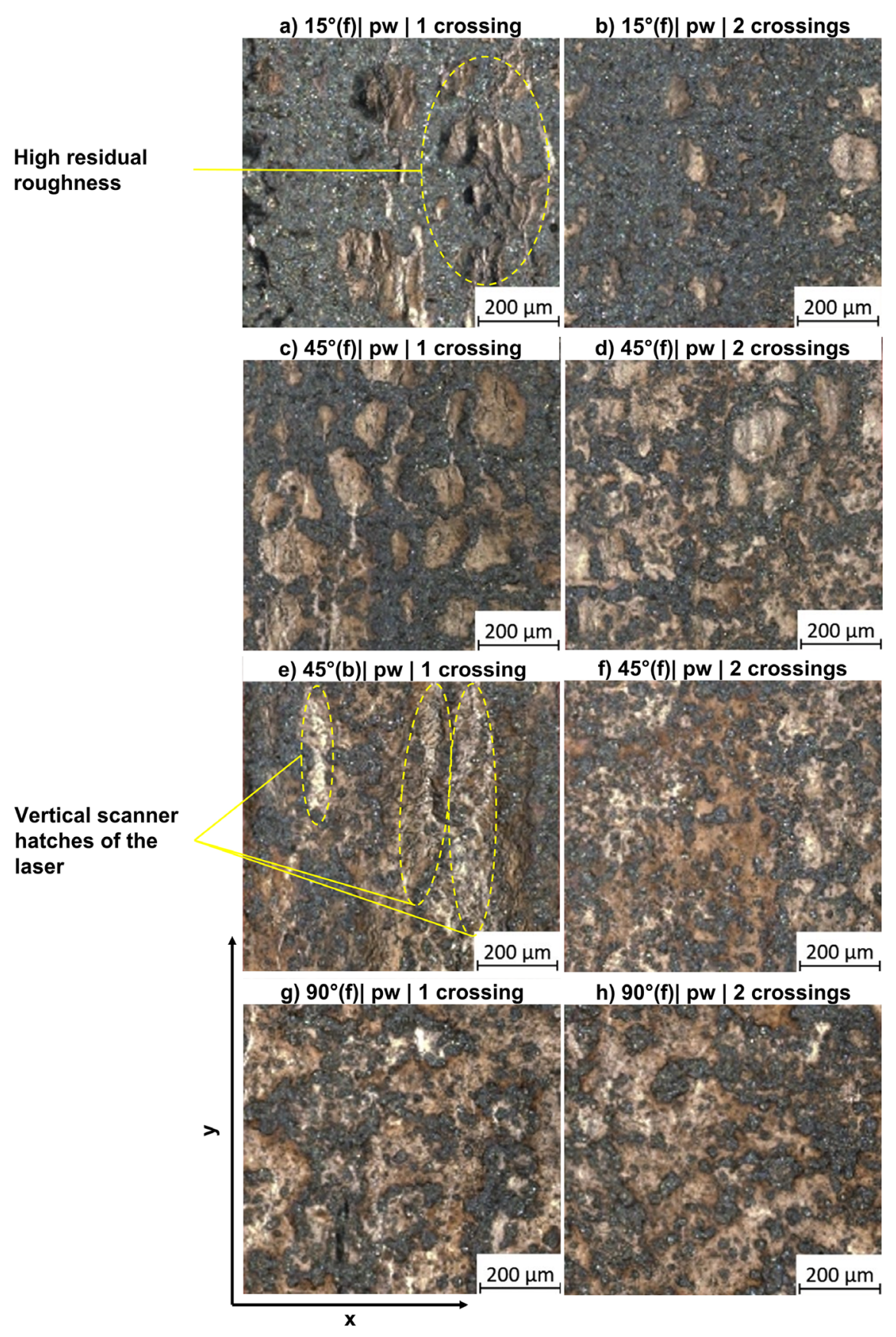

Figure 13. Microscopic pictures of the surface in selected areas in the middle of the polishing fields after pulsed mode laser polishing with one and two polishing passes depending on the fabrication angles, (a)/(b) $15^{\circ}$ (frontside), (c)/(d) $45^{\circ}$ (frontside), (e)/(f) $45^{\circ}$ (backside) and $(\mathrm{g}) /(\mathrm{h}) 90^{\circ}$ (frontside) with the scanner pendulum movement in y-direction and the axis movement in $\mathrm{x}$-direction.

Figure 14 shows the macroscopic 3D topography of the test fields after laser polishing with pulsed radiation. It becomes clear, that the short and medium surface structures, caused by the welding tracks of the powder bed fusion process, melt pool or spatter material accumulations and the stairs of the inclined additive fabrication angle are flattened compared to the initial structures at the bor- 
der of the images. A comprehensive view of the polishing field shows that long wavelength structures and height variations are still present. Thus, after laser polishing, a waviness with structure wavelengths above the cut-off wavelength of the roughness spectrum are still visible. Additionally, especially at $45^{\circ}$ degree backside, at the turning points of the scanner beam deflection a surface super elevation by material accumulation occurs.

When comparing the residual microscopic structures at the centre of the polishing fields the residual roughness structures in the range of $50-100 \mu \mathrm{m}$ structure wavelength get visible (Figure 15). In comparison to the other polishing fields, the polishing field with $90^{\circ}$ degree fabrication angle differs with its flat global surface profile.

\subsection{Continuous Mode Laser Polishing}

In contrast to the investigations on pulsed mode laser polishing, continuous mode polishing was done with only one crossing. Therefore two different axis velocities $\mathrm{v}_{\mathrm{f}}=250 \mathrm{~mm} / \mathrm{min}$ with a track overlap of $92.1 \%$ and $\mathrm{v}_{\mathrm{f}}=350 \mathrm{~mm} / \mathrm{min}$ with a track overlap of $89.0 \%$ and the parameters mentioned in Table 1 were used. The achieved surface roughness Ra, measured in sample vertical direction (SVD) (in SVD) and perpendicular (perpendicular SVD) under varying fabrication angles are given in Figure 16.

a)

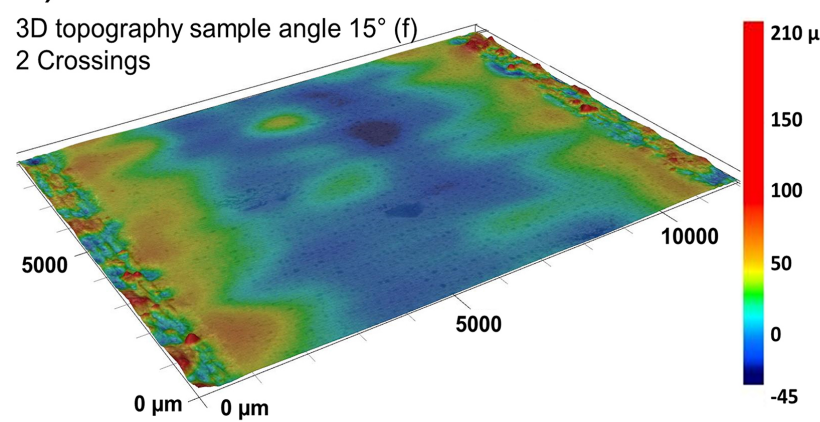

b)

3D topography sample angle $90^{\circ}$ (f)

2 Crossings

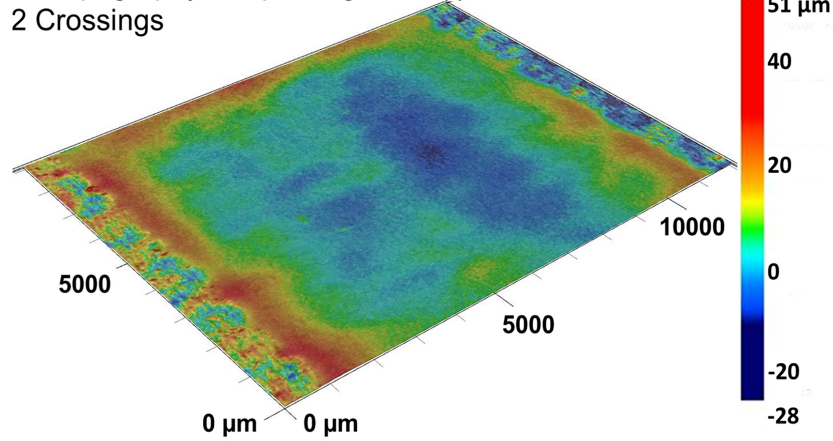

c)

$3 \mathrm{D}$ topography sample angle $45^{\circ}(\mathrm{f})$

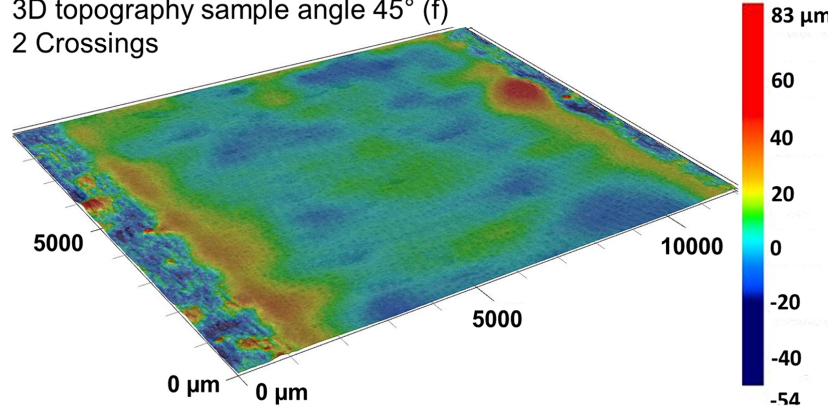

d)

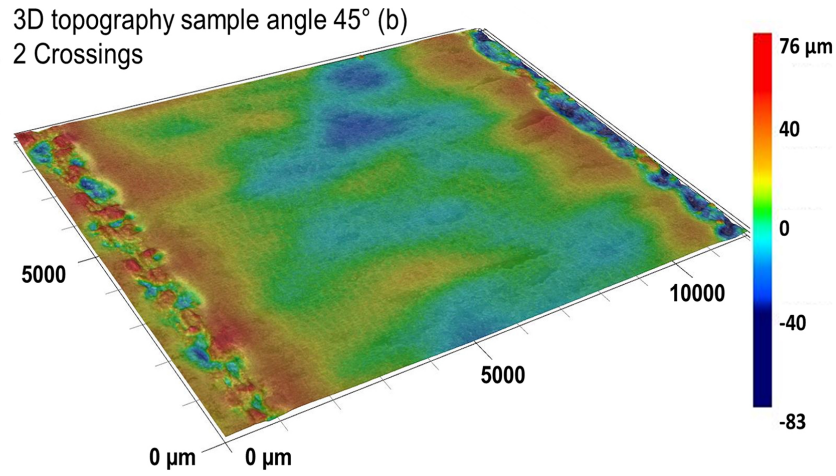

Figure 14. Qualitative comparison of the achieved surface topography during pulsed laser polishing with two crossings (a) fabrication angle $15^{\circ}$ (b) fabrication angle $90^{\circ}$, (c) fabrication angle $45^{\circ}$, (d) fabrication angle $45^{\circ}$ Backside. 
a)

$3 \mathrm{D}$ topography sample angle $15^{\circ}(\mathrm{f})$

2 Crossings

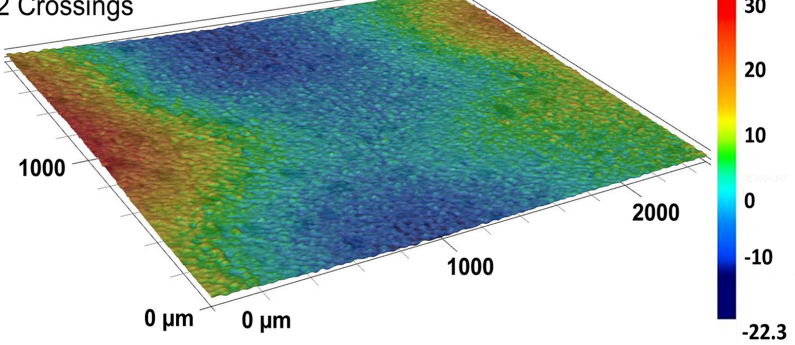

b)

$3 \mathrm{D}$ topography sample angle $90^{\circ}$ (f)

2 Crossings

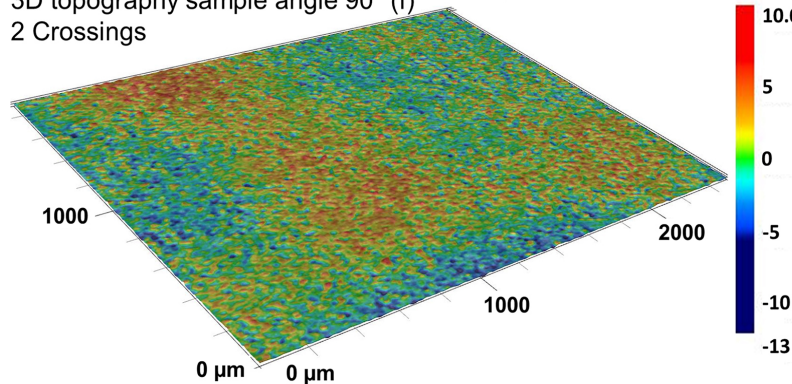

c)

$34.4 \mu \mathrm{m} 3 \mathrm{D}$ topography sample angle $45^{\circ}$ (f)

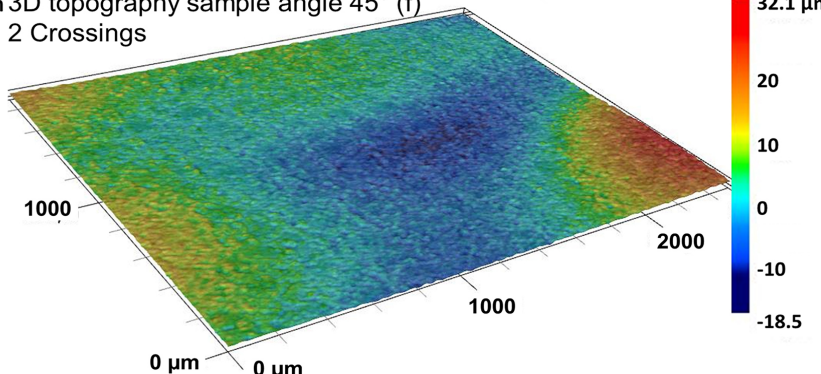

d)

$3 \mathrm{D}$ topography sample angle $45^{\circ}$ (b)

2 Crossings

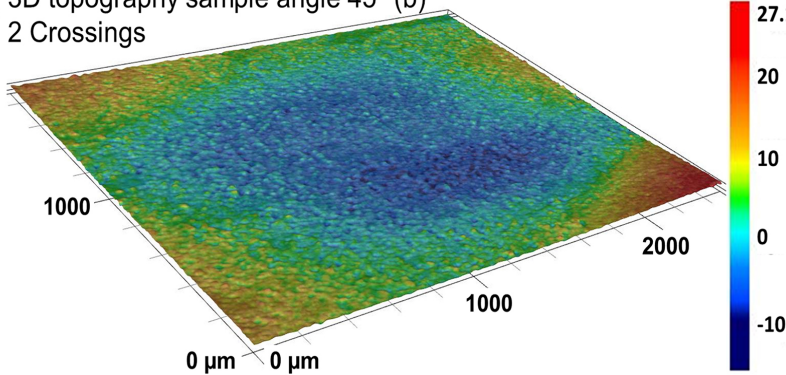

Figure 15. Microscopic differences at a 40-fold magnification of the achieved surface topography after pulsed laser polishing with two crossings at the centre of the polishing fields (a) fabrication angle $15^{\circ}$ frontside, (b) fabrication angle $90^{\circ}$ fronside, (c) fabrication angle $45^{\circ}$ frontside, (d) fabrication angle $45^{\circ}$ backside.

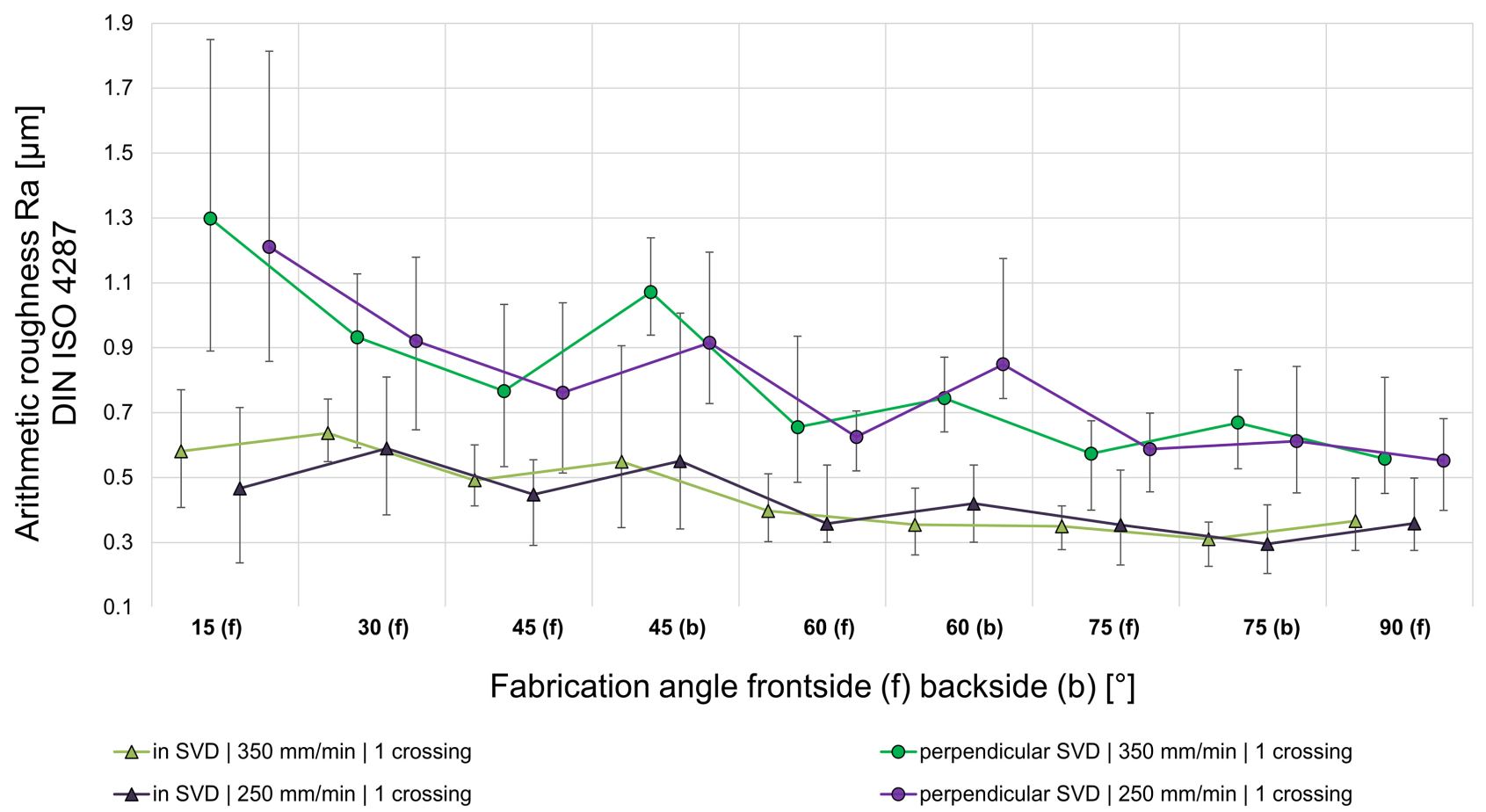

Figure 16. Arithmetic roughness Ra according to DIN ISO 4287 measured in and perpendicular to the sample vertical direction (SVD) under varying fabrication angle with continuous laser radiation for one crossing with an axis velocity $\mathrm{vf}_{\mathrm{f}}$ of $250 \mathrm{~mm} / \mathrm{min}$ and $350 \mathrm{~mm} / \mathrm{min}$. 
Figure 16 shows that the roughness of the laser polished samples is in a range between $1.3 \mu \mathrm{m}$ and $0.3 \mu \mathrm{m}$. Similar to the polishing with pulsed mode, the polishing with continuous mode shows, that the achieved surface roughness is reduced with an increasing fabrication angle. At a fabrication angle of $15^{\circ}(\mathrm{f})$, a roughness of e.g. $\mathrm{Ra}=0.47 \mu \mathrm{m}\left(\mathrm{v}_{\mathrm{f}}=250 \mathrm{~mm} / \mathrm{min}\right.$, in SVD) was achieved. By increasing the fabrication angle up to $90^{\circ}$ the roughness was reduced to a minimum of $\mathrm{Ra}=0.36 \mu \mathrm{m}\left(\mathrm{v}_{\mathrm{f}}=250 \mathrm{~mm} / \mathrm{min}\right.$, in SVD). Also similar to the polishing with pulsed mode a higher roughness measured perpendicular to SVD (e.g. $30^{\circ}$ (f) $\left.\mid \mathrm{v}_{\mathrm{f}}=250 \mathrm{~mm} / \mathrm{min}: \mathrm{Ra}=0.92 \mu \mathrm{m}\right)$ compared to the measurement in $\operatorname{SVD}\left(30^{\circ}\right.$ (f) $\mid \mathrm{v}_{\mathrm{f}}=250 \mathrm{~mm} / \mathrm{min}: \mathrm{Ra}=0.59 \mu \mathrm{m}$ ) exists. Additionally the roughness variation in the test fields, shown by the scattering bars, is significant higher perpendicular to SVD and correlates to the variations at the initial roughness after 3D printing. A reduced axis velocity of $250 \mathrm{~mm} / \mathrm{min}$ at continuous laser polishing offers no steady improvement over all fabrication orientations. A higher roughness after laser polishing exists on the overhanging backsides surfaces for the fabrication angles $45^{\circ}, 60^{\circ}$ and $70^{\circ}$, compared to the frontsides, with the exception for an axis velocity $\mathrm{v}_{\mathrm{f}}$ of $350 \mathrm{~mm} / \mathrm{min}$ measured in SVD at $60^{\circ}$ and for both axis velocities measured in SVD at $75^{\circ}$.

Similar to the investigations with pulsed mode polishing, the percental roughness reduction for polishing with continuous laser radiation, measured in and perpendicular to the sample vertical direction (SVD) is given in Figure 17.

Figure 17 shows that a higher average roughness reduction over all fabrication angles is achieved in SVD (average-reduction $=96.4 \%$ at $\mathrm{v}_{\mathrm{f}}=250 \mathrm{~mm} / \mathrm{min}$ ) compared with perpendicular to SVD (average-reduction $=93.6 \%$ at $\mathrm{v}_{\mathrm{f}}=250$ $\mathrm{mm} / \mathrm{min}$ ). By measuring the roughness in sample vertical direction, the relative

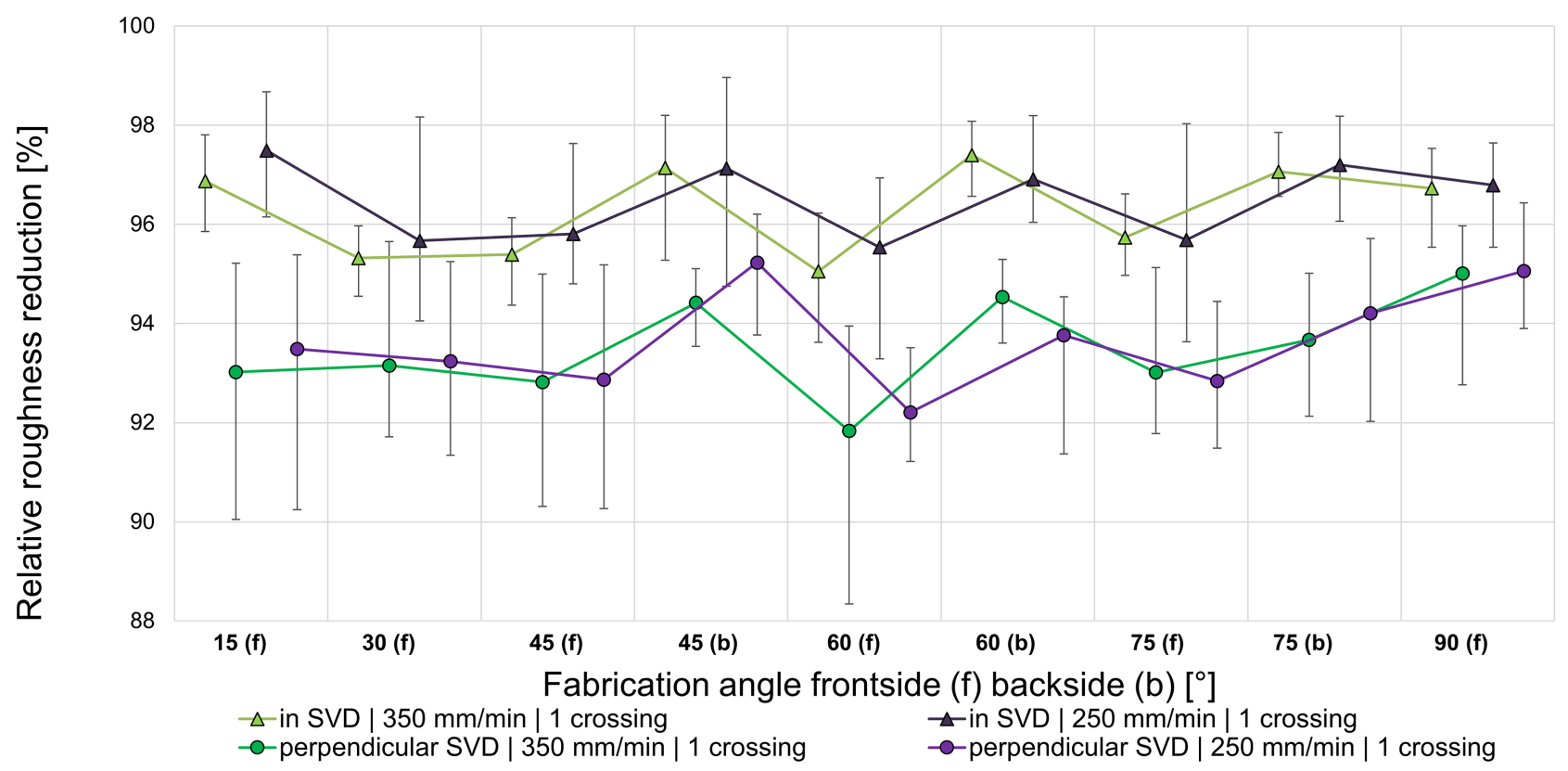

Figure 17. Relative roughness reduction in percent, measured in and perpendicular to the sample vertical direction (SVD) for an axis velocity of $\mathrm{v}_{\mathrm{f}}=250 \mathrm{~mm} / \mathrm{min}$ and $\mathrm{v}_{\mathrm{f}}=350 \mathrm{~mm} / \mathrm{min}$ with continuous radiation under varying the fabrication angle. 
roughness reduction is more or less independent of the respective fabrication angles. Also similar to the results with pulsed mode is that the respective backsides of the samples over all construction angles have a higher roughness reduction for both measuring directions compared to roughness reduction on the frontsides.

Perpendicular to SVD the resulting surface roughness reduction rates at $90^{\circ}$ of approximately $95 \%$, is significant higher compared to the further frontside surfaces of the samples with smaller fabrication angles.

When analyzing the partial roughness over the structural wavelength ranges of the polished surface (Figure 18), it becomes clear that the main differences between the individual fabrication angles are in the medium to long wavelength range. When observing the spectral roughness in the range of $2.5-3.9 \mu \mathrm{m}$, the roughness variation amounts $50 \%$ from $\mathrm{Ra}=0.014 \mu \mathrm{m}$ to $\mathrm{Ra}=0.028 \mu \mathrm{m}$. At the medium structure wavelength the polished surface of the specimen with $30^{\circ}$ degree fabrication angle exhibits der greatest residual roughness, e.g. at 15.6 - 31.25 $\mu \mathrm{m} \mathrm{Ra}=0.22 \mu \mathrm{m}$. Larger differences can be found in the longer wavelength above $125 \mu \mathrm{m}$, where there is a big gap between the fabrication angles $15^{\circ}$ degree and $45^{\circ}$ degree backside and the further fabrication angles. For example at the range between $500-800 \mu \mathrm{m}$ the partial arithmetic surface roughness $\mathrm{Ra}$ is with $0.78 \mu \mathrm{m}\left(45^{\circ}\right.$ backside) more than three times as high compared to $75^{\circ}$ (f) with $\mathrm{Ra}=0.22 \mu \mathrm{m}$. Compared to the pulsed laser mode, smaller deviations are detected, which results in a slightly better process stability in the continuous laser mode.

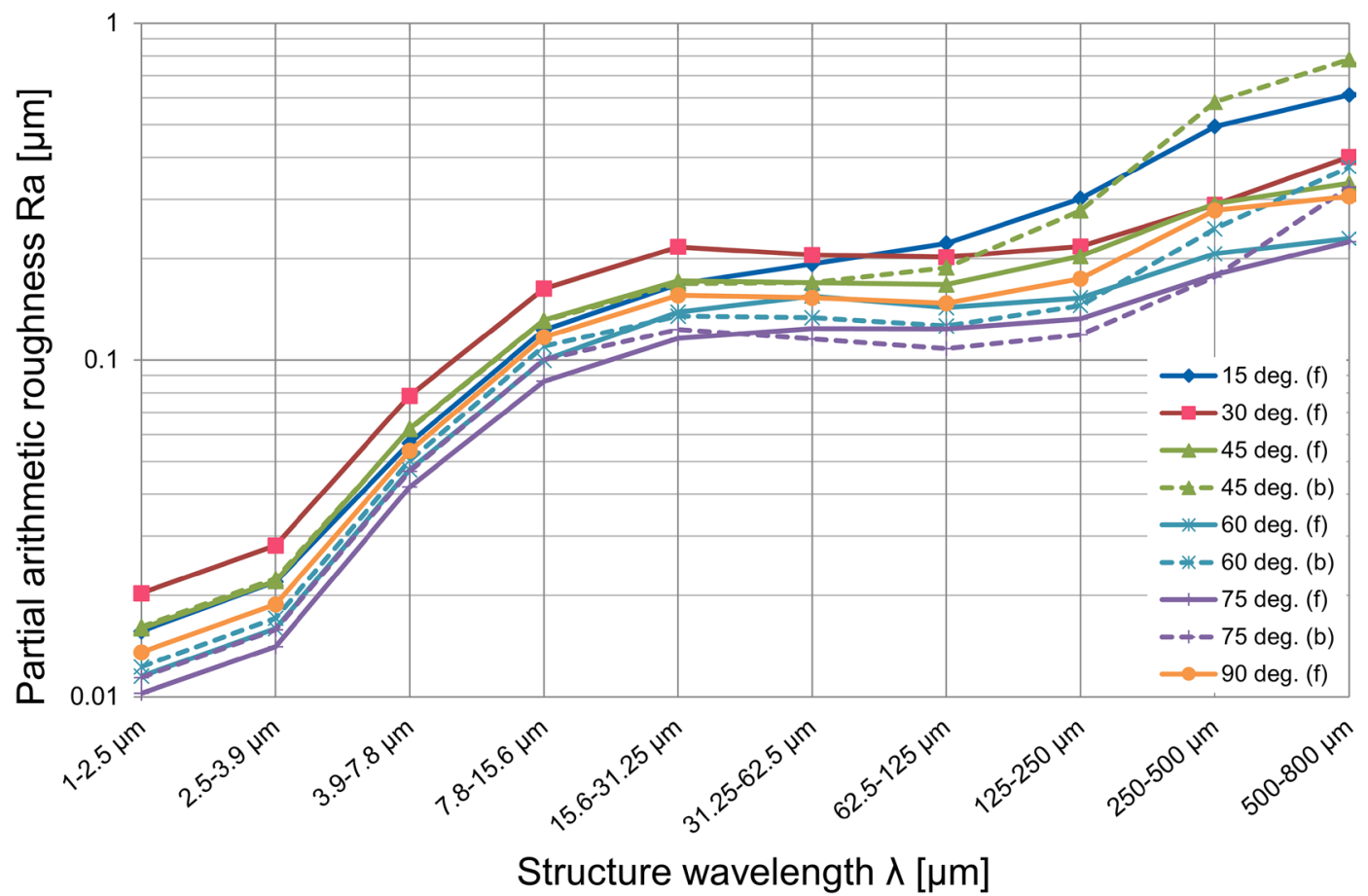

Figure 18. Partial roughness over structure wavelength with continuous mode polishing: Influence of the fabrication angle polished in SVD for one crossing frontside (f), backside (b). 
At continuous mode polishing the differences in the surfaces appearance and surface structures between the different fabrication angles under consideration of the used axis velocities of $\mathrm{v}_{\mathrm{f}}=250 \mathrm{~mm} / \mathrm{min}$ and $\mathrm{v}_{\mathrm{f}}=350 \mathrm{~mm} / \mathrm{min}$ are displayed in the microscopic images of Figure 19. Similar to pulsed mode polishing the remolten surface layer exhibits encrusted surfaces topography (grey surface

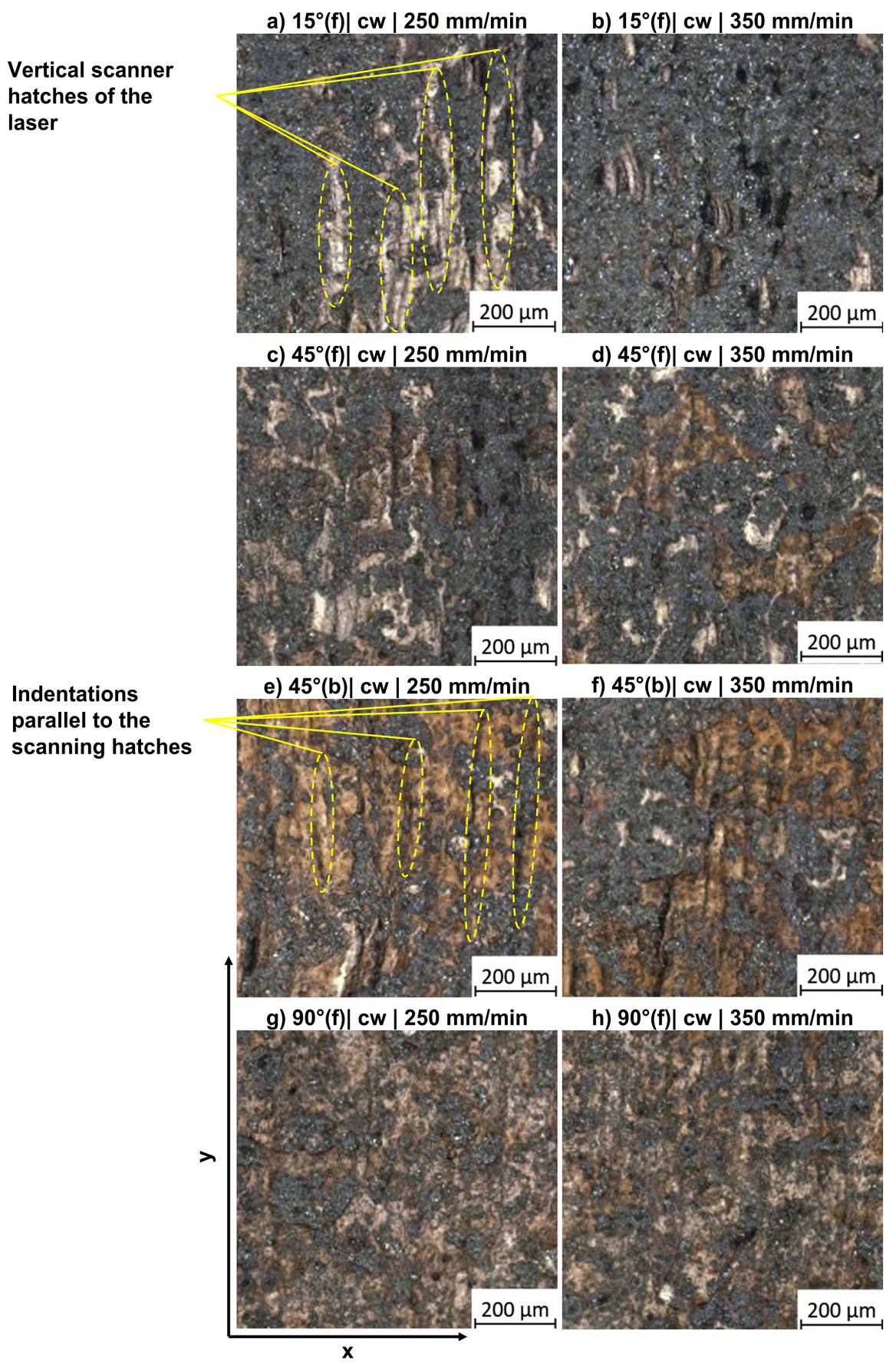

Figure 19. Microscopic surface pictures after continuous mode laser polishing with an axis velocity of $\mathrm{v}_{\mathrm{f}}=250 \mathrm{~mm} / \mathrm{min}$ and $\mathrm{v}_{\mathrm{f}}=350 \mathrm{~mm} / \mathrm{min}$ depending on fabrication angle, (a) $15^{\circ}$ (f), (b) $45^{\circ}$ (f), (c) $90^{\circ}$ (f), (d) 45 (b) with the movement of the scanner pendulum $\mathrm{y}$ and the axis velocity $\mathrm{x}$. 
structures), which decrease with an increasing fabrication angle. The resulting Surface layers after surface remelting of additive manufactured AlSi10Mg, processed under comparable process atmosphere, were investigated by Schanz by means of SEM and EDX [38], where a higher concentration of Oxygen and Magnesium was detected. Linear indentations parallel to the scanning hatches, respective to the scanner pendulum direction (y-direction) are clearly visible at small fabrication angles. They disappear at vertical built parts (Figure 19(g) and Figure 19(h)).

The macroscopic 3D topography after laser polishing with continuous laser radiation is shown in Figure 20. In comparison to the polishing field at the fabrication angles $45^{\circ}$ and $90^{\circ}$ frontside the surface at $15^{\circ}$ degree has a unevenness with large areal elevations and depressions. At $45^{\circ}$ backside waviness with shorter wavelength and smaller areal expansion can be seen. While at polishing with pulsed laser radiation a material accumulation at the turning points occurs, continuous laser radiation achieves an almost homogenies and flat transition between the remolten surface layer and the initial surface.

At a 40 -fold magnification at the center of the polishing fields the residual microscopic roughness structures in the range of $50-100 \mu \mathrm{m}$ structure wavelength get visible (Figure 21). When comparing maximum height variation, the surface at $45^{\circ}$ degree frontside and $90^{\circ}$ are significant smother.

a)

$3 \mathrm{D}$ topography sample angle $15^{\circ}$ (f) $v_{\mathrm{f}}=250 \mathrm{~mm} / \mathrm{min}$

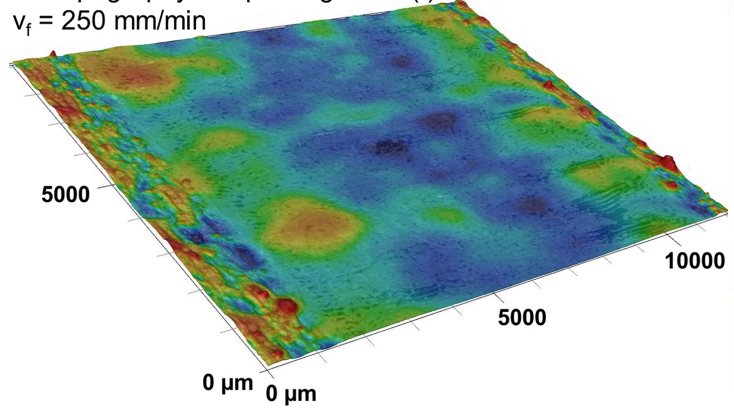

b)

$3 \mathrm{D}$ topography sample angle $90^{\circ}$ (f) $\mathrm{v}_{\mathrm{f}}=250 \mathrm{~mm} / \mathrm{min}$

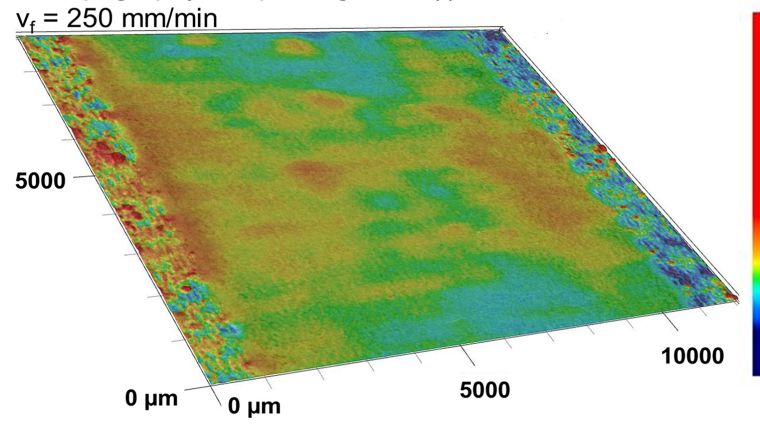

C)

3D topography sample angle $45^{\circ}$ (f)

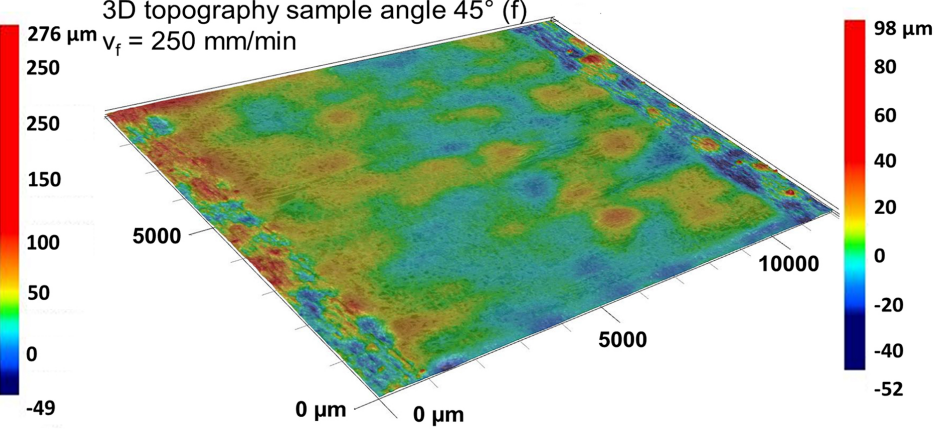

d)

3D topography sample angle $45^{\circ}$ (b)
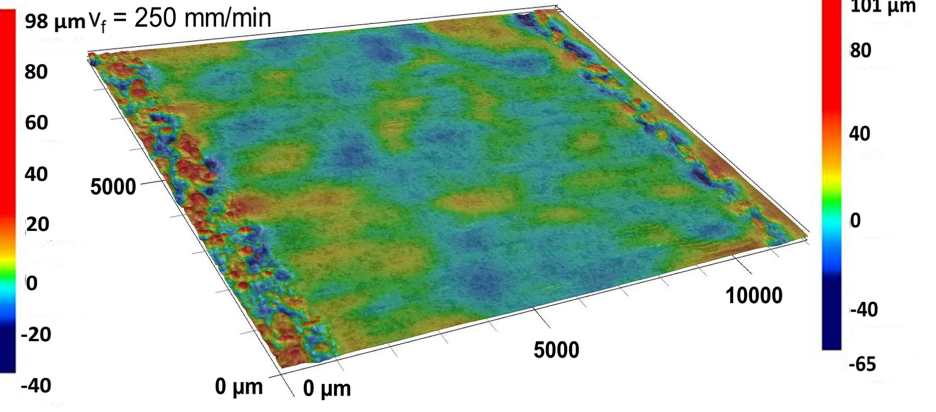

Figure 20. Qualitative comparison of the achieved surface topography during continuous laser polishing with an axis velocity of $\mathrm{v}_{\mathrm{f}}$ $=250 \mathrm{~mm} / \mathrm{min}$ (a) fabrication angle $15^{\circ}$ frontside (b) fabrication angle $90^{\circ}$ frontside, (c) fabrication angle $45^{\circ}$ frontside, (d) fabrication angle $45^{\circ}$ backside. 
a)

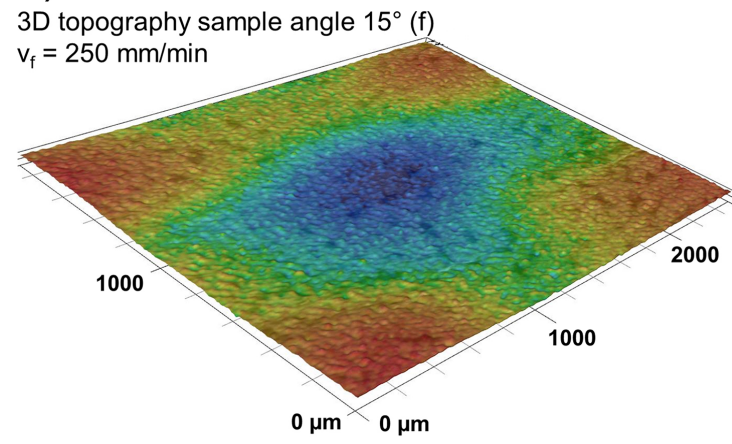

b)

$3 \mathrm{D}$ topography sample angle $90^{\circ}(\mathrm{f})$

$\mathrm{v}_{\mathrm{f}}=250 \mathrm{~mm} / \mathrm{min}$

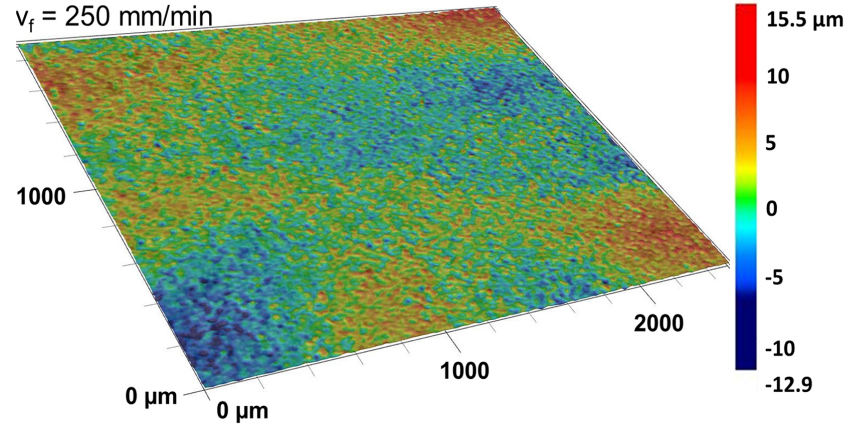

c)

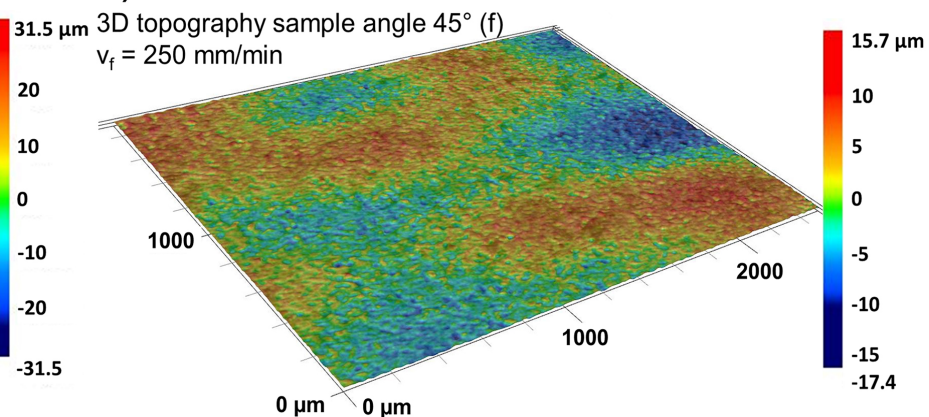

d)

3D topography sample angle $45^{\circ}(\mathrm{b})$

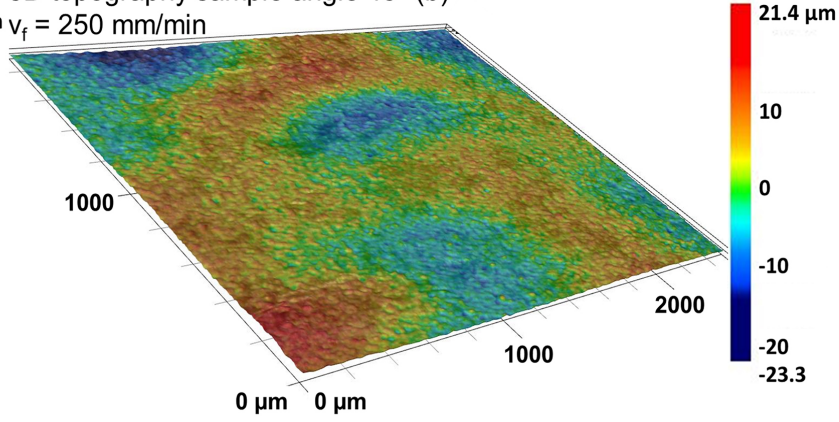

Figure 21. Microscopic differences at a 40-fold magnification of the achieved surface topography after continuous laser polishing with an axis velocity of $250 \mathrm{~mm} / \mathrm{min}$ at the centre of the polishing field (a) fabrication angle $15^{\circ}$ frontside, (b) fabrication angle $90^{\circ}$ frontside, (c) fabrication angle $45^{\circ}$ frontside, (d) fabrication angle $45^{\circ}$ backside.

\subsection{Fundamental Process Dependencies and Differences between the Laser Operation Modes}

Laser polishing with pulsed and continuous laser radiation on varying initial surface structures after 3D-printing, generated by varying the fabrication angle, was investigated. The presented results have shown that the roughness measured in the sample vertical direction is consistently lower than the roughness measured perpendicular to the sample vertical direction. This can be explained by a better smoothing capacity respectively by a higher volume distribution within the scanner axis, because the movement of the scanner is a multiple times higher compared to the axis movement (see also Figure 4). The higher reduction of roughness shown on the backsides can be caused by their structure, which is different from the structures of the front sides. Considering the initial roughness after the Fourier transformation (Figure 7), large differences in the longer wavelength spectrum can be observed. For example, the initial roughness in the wavelength spectrum of 156.3 to $312.5 \mu \mathrm{m}$ for a fabrication angle of $30^{\circ}$ (f) has a value of $\mathrm{Ra}=2.58 \mu \mathrm{m}$ and an achieved roughness by pulsed polishing of $\mathrm{Ra}=0.2$ $\mu \mathrm{m}$ (spectrum $125-250 \mu \mathrm{m}$ ), whilst the initial roughness in the same spectrum for a fabrication angle of $45^{\circ}$ (b) is $\mathrm{Ra}=6.72 \mu \mathrm{m}$ and the achieved roughness by pulsed polishing is as well $\mathrm{Ra}=0.2 \mu \mathrm{m}$ (spectrum $125-250 \mu \mathrm{m}$ ). The same can be observed for polishing with continuous wave where the initial roughness at a 
spectrum of $156.3-312.5 \mu \mathrm{m}$ for a fabrication angle of 90 (f) is $\mathrm{Ra}=5.3 \mu \mathrm{m}$ and the achieved roughness at a spectrum of $125-250 \mu \mathrm{m}$ is $\mathrm{Ra}=0.17 \mu \mathrm{m}$ compared to the initial roughness of a fabrication angle of $60^{\circ}$ (b) with an initial roughness at a spectrum of $156.3-312.5 \mu \mathrm{m}$ of $\mathrm{Ra}=5.5 \mu \mathrm{m}$ and an achieved roughness at a spectrum of 125 - $250 \mu \mathrm{m}$ of $\mathrm{Ra} 0.15 \mu \mathrm{m}$.

By applying the achieved arithmetic roughness $\mathrm{Ra}$ after laser polishing at both laser operation modes relating to the initial surface roughness after 3D-printing, the roughness reduction rate can be compared. Following Figure 22, the collected measurement data are displayed of both laser operation modes, measured in sample vertical direction (SVD).

When looking at the dependency between the initial surface roughness and the surface quality after laser polishing, measured in SVD (Figure 22), the differences in the resulting roughness after laser polishing increases with an increasing initial roughness. Thus the polished surface at an initial roughness $\mathrm{Ra}$ approximately $8 \mu \mathrm{m}$ changes between $\mathrm{Ra}=0.25 \mu \mathrm{m}-0.39 \mu \mathrm{m}$ in contrast to $\mathrm{Ra}=$ $0.33 \mu \mathrm{m}-0.64 \mu \mathrm{m}$ at an initial roughness in the range of $13.6 \mu \mathrm{m}$. Moreover, a higher smoothing effect can be achieved on the backside surfaces at both laser operation modes. Hence, a higher amount of data dots on the backsides (b) (9 pieces) is above a roughness reduction of $>97 \%$ compared to the data dots on the frontsides ( $\mathrm{f}$ ( 4 pieces). With the exception of the fabrication angle of 15 degrees, pulsed laser polishing with two crossings achieves the lowest roughness over all fabrication angles.

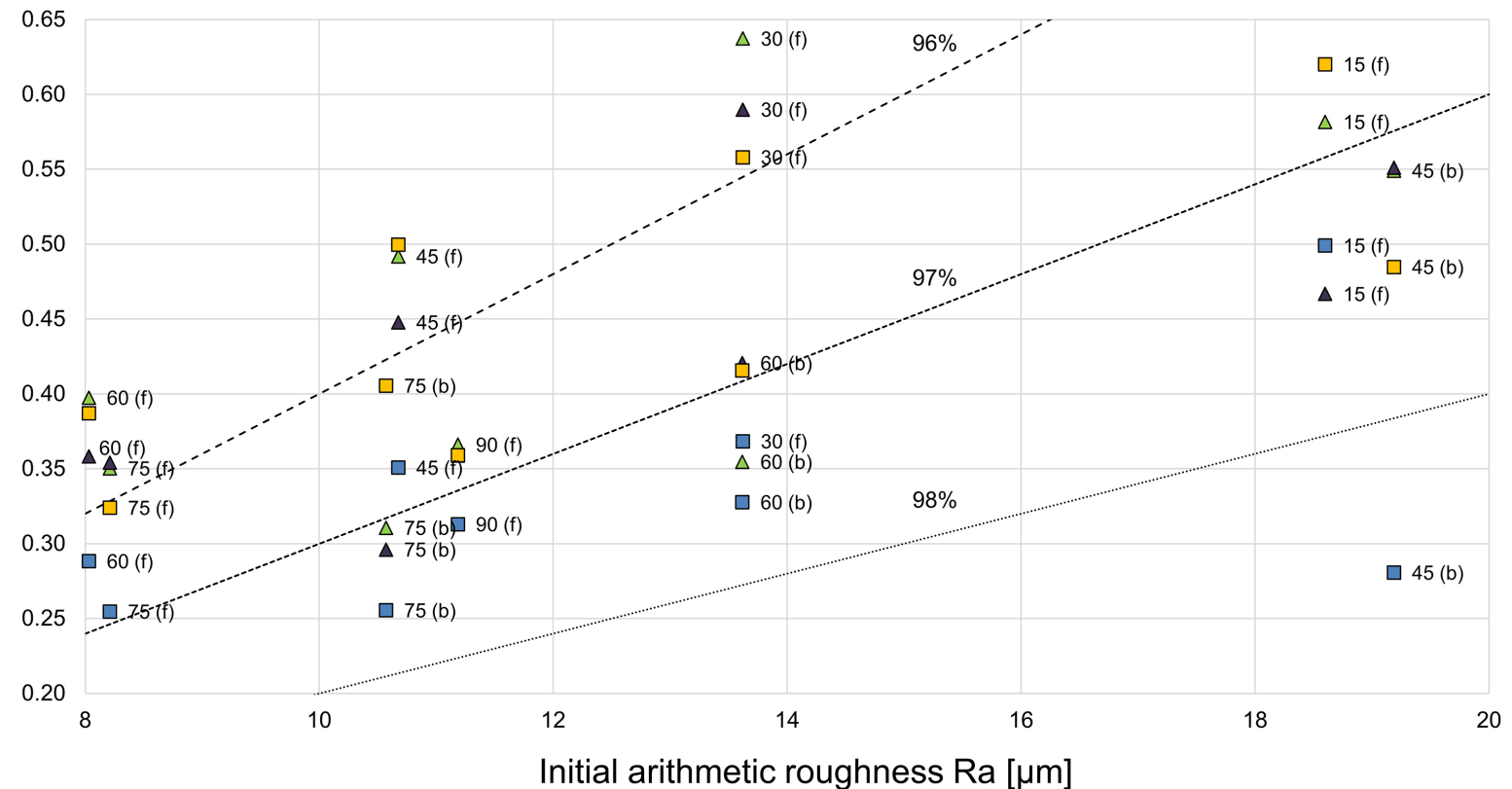

$\Delta \mathrm{CW}$ | in SVD | $350 \mathrm{~mm} / \mathrm{min} \mid 1$ crossing $\Delta \mathrm{cW} \mid$ in SVD $|250 \mathrm{~mm} / \mathrm{min}| 1$ crossing $\square \mathrm{pw} \mid$ in SVD $\mid 1$ crossing $\square \mathrm{pw} \mid$ in SVD $2 \mathrm{crossings}$

Figure 22. Achieved arithmetic roughness Ra after laser polishing depending on the initial Roughness Ra, measured in sample vertical direction (SVD) according to DIN ISO 4287 for one and two crossings at pulsed laser radiation and for an axis velocity of $250 \mathrm{~mm} / \mathrm{min}$ and $350 \mathrm{~mm} / \mathrm{min}$ for continuous laser radiation under varying fabrication angle. Lines of constant relative roughness reductions at $96 \%, 97 \%$ and $98 \%$ are incorporated. 
Table 2. Comparison of the achieved arithmetic roughness $\mathrm{Ra}$ and partial roughness of structure wavelength ranges, measured in SVD, with one crossings and an axis velocity at continuous mode laser polishing of $250 \mathrm{~mm} / \mathrm{min}$.

\begin{tabular}{ccccc}
\hline & $\begin{array}{c}\text { Roughness } \\
\mathbf{R}_{\mathrm{a}, \mathrm{cw}} / \mathbf{R}_{\mathrm{a}, \mathrm{pw}}\end{array}$ & \multicolumn{3}{c}{$\begin{array}{c}\text { Partial roughness } \\
\mathbf{R}_{\mathrm{a}, \mathrm{cw}} / \mathbf{R}_{\mathrm{a}, \mathrm{pw}}\end{array}$} \\
\hline Fabrication Angle & $\lambda=1 \mu \mathrm{m}-800 \mu \mathrm{m}$ & $\lambda=2.5-3.9 \mu \mathrm{m}$ & $\lambda=31.25-62.5 \mu \mathrm{m}$ & $\lambda=500-800 \mu \mathrm{m}$ \\
\hline \multirow{2}{*}{$15^{\circ}$ (f) } & $0.467 \mu \mathrm{m}$ & $0.022 \mu \mathrm{m}$ & $0.193 \mu \mathrm{m}$ & $0.612 \mu \mathrm{m}$ \\
& $0.621 \mu \mathrm{m}$ & $0.021 \mu \mathrm{m}$ & $0.170 \mu \mathrm{m}$ & $0.616 \mu \mathrm{m}$ \\
$75^{\circ}(\mathrm{f})$ & $0.354 \mu \mathrm{m}$ & $0.014 \mu \mathrm{m}$ & $0.116 \mu \mathrm{m}$ & $0.225 \mu \mathrm{m}$ \\
& $0.324 \mu \mathrm{m}$ & $0.018 \mu \mathrm{m}$ & $0.120 \mu \mathrm{m}$ & $0.311 \mu \mathrm{m}$ \\
$45^{\circ}$ (b) & $0.551 \mu \mathrm{m}$ & $0.022 \mu \mathrm{m}$ & $0.170 \mu \mathrm{m}$ & $0.782 \mu \mathrm{m}$ \\
& $0.485 \mu \mathrm{m}$ & $0.016 \mu \mathrm{m}$ & $0.098 \mu \mathrm{m}$ & $0.498 \mu \mathrm{m}$ \\
\hline
\end{tabular}

When comparing the resulting arithmetic surface roughness $\mathrm{Ra}$ and the partial roughness $\mathrm{Ra}$ on small, medium and long structure wavelengths after one crossing, only small differences (for example for $15^{\circ}$ (f) $0.004 \mu \mathrm{m}$ at a wavelength between 500 and $800 \mu \mathrm{m}$ ) at the frontside surfaces with fabrication angles $15^{\circ}$ and $75^{\circ}$ exists (Table 2). At the backside surface with 45 degree fabrication angle and the highest initial roughness, the pulsed mode polishing achieves a better surface quality at the medium and long structure wavelength.

\section{Conclusions}

The Laser polishing of AlSi10Mg SLM parts with continuous and pulsed laser radiation under varying the fabrication angle was investigated. By changing the fabrication angle between $15^{\circ}$ and vertical built specimens $\left(90^{\circ}\right.$ degree fabrication angle), the initial arithmetic roughness varies between $19.2 \mu \mathrm{m}$ and $8.0 \mu \mathrm{m}$. With increasing fabrication angle the roughness decreases till $60^{\circ}$ degree. The overhanging backside surfaces exhibit a significant higher roughness, which is more than doubled compared to the frontside at a fabrication angle of $45^{\circ}$ degree. Additionally the analyzed roughness composition within the Fourier transformation and 3D Topography measurements shows, that the main roughness components are above structure wavelength of $30 \mu \mathrm{m}$.

Pulsed mode and continuous mode polishing deliver a major relative roughness reduction up to $98.5 \%$. The achievable final roughness after laser polishing at both laser operation modes directly depends on the initial surface quality. With decreasing initial roughness the roughness after laser polishing is decreasing. The relative roughness reduction over all investigated fabrication angles for one crossing, measured in sample vertical direction, respectively the scanner pendulum movement is almost constant and varies between $95.2 \%$ and $97.5 \%$ at the pulsed laser mode and $95.0 \%-97.5 \%$ at the continuous laser mode. Thus the smoothing ability, given by the relative roughness reduction, does not decline with an increasing initial roughness, which is an important fact for surface treatment of complex 3D freeform surfaces with steadily varying fabrication angle. A 
constantly lower relative roughness reduction over all fabrication angles at both laser operation modes is measured perpendicular to SVD. When comparing the front- and backside surface of the samples a constantly higher relative roughness reduction rate at both laser operation modes is achieved on the overhanging backside surfaces.

The analyzation of the residual partial roughness over structure wavelength shows, that the achieved surface quality at small structure wavelength has only small differences between the sample vertical directions. Comparable to the initial surface, the main residual roughness is found in the medium and long structure wavelength. At the medium structure wavelengths caused at small fabrication angles by the stairs effect, at pulsed mode polishing the roughness is noticeable higher. The residual partial roughness at long structure wavelength above $125 \mu \mathrm{m}$ is directly depending on the initial roughness structures.

The 3D topography measurement shows, that the polishing fields exhibit an additional wide spread waviness. While continuous laser mode polishing exhibit a flat transition between the remolten surface layer and the initial surface, pulsed mode polishing leads to a material accumulation at the turning points of the scanner pendulum movement.

\section{Acknowledgements and Funding}

The authors would like to thank Michael Sedlmajer from the Institute of Virtual Product Development at Aalen University for manufacturing the samples.

We acknowledge support by the Federal Ministry of Economics and Technology German, program “ZIM” (Zentrales Innovationsprogramm Mittelstand), (project "3D-LaPol") and German Federal Ministry of Education and Research, program "FH-Impuls" (AddFunK, grant No. 03FH4I04IA) and "FH-Invest" (Project FlexLight 4.0, Grant No. 13FH114N6).

\section{Conflicts of Interest}

The authors declare no conflicts of interest regarding the publication of this paper.

\section{References}

[1] Kempen, K., Thijs, L., van Humbeeck, J. and Kruth, J.-P. (2012) Mechanical Properties of AlSi10Mg Produced by Selective Laser Melting. Physics Procedia, 39, 439-446. https://doi.org/10.1016/j.phpro.2012.10.059

[2] Mower, T.M. and. Long, M.J. (2016) Mechanical Behavior of Additive Manufactured, Powder-Bed Laser-Fused Materials. Materials Science and Engineering. A, 651, 198-213. https://doi.org/10.1016/j.msea.2015.10.068

[3] Hitzler, L., Janousch, C., Schanz, J., Merkel, M., Mack, F. and Öchsner, A. (2016) Non-Destructive Evaluation of AlSi10Mg Prismatic Samples Generated by Selective Laser Melting: Influence of Manufacturing Conditions. Materials Science \& Engineering Technology, 47, 564-581. https://doi.org/10.1002/mawe.201600532

[4] Yasa, E. and Kruth, J.-P. (2011) Microstructural Investigation of Selective Laser Melt- 
ing 316L Stainless Steel Parts Exposed to Laser Re-Melting. Procedia Engineering, 19, 389-395. https://doi.org/10.1016/j.proeng.2011.11.130

[5] van Hooreweder, B., Lietaert, K., Neirinck, B., Lippiatt, N. and Wevers, M. (2017) CoCr F75 Scaffolds Produced by Additive Manufacturing: Influence of Chemical Etching on Powder Removal and Mechanical Performance. Journal of the Mechanical Behavior of Biomedical Materials, 70, 60-67.

https://doi.org/10.1016/j.jmbbm.2017.03.017

[6] Hitzler, L., Janousch, C., Schanz, J., Merkel, M., Heine, B., Mack, F., Hall, W. and Öchsner, A. (2017) Direction and Location Dependency of Selective Laser Melted AlSi10Mg Specimens. Journal of Materials Processing Technology, 243, 48-61. https://doi.org/10.1016/j.jmatprotec.2016.11.029

[7] Ch, S.R., Raja, A., Nadig, P., Jayaganthan, R. and. Vasa, N.J. (2019) Influence of Working Environment and Built Orientation on the Tensile Properties of Selective Laser Melted AlSi10Mg alloy. Materials Science and Engineering A, 750, 141-151. https://doi.org/10.1016/j.msea.2019.01.103

[8] Yang, T., Liu, T., Liao, W., MacDonald, E., Wei, H., Chen, X. and Jiang, L. (2019) The Influence of Process Parameters on Vertical Surface Roughness of the AlSi10Mg Parts Fabricated by Selective Laser Melting. Journal of Materials Processing Technology, 266, 26-36. https://doi.org/10.1016/j.jmatprotec.2018.10.015

[9] Calignano, F., Manfredi, D., Ambrosio, E. P., Iuliano, L. and Fino, P. (2013) Influence of Process Parameters on Surface Roughness of Aluminum Parts Produced by DMLS. The International Journal of Advanced Manufacturing Technology, 67, 2743 2751. https://doi.org/10.1007/s00170-012-4688-9

[10] Dewi, H.S. and Volpp, J. (2020) Impact of Laser Beam Oscillation Strategies on Surface Treatment of Microalloyed Steel. Journal of Laser Applications, 32, Article No. 42006. https://doi.org/10.2351/7.0000196

[11] Yasa, E. and Kruth, J.-P. (2014) Application of Laser Remelting on Selective Laser Melting Parts. Advances in Production Engineering \& Management, 6, 259-270

[12] Gebhardt, A., Hötter, J.-S. and Ziebura, D. (2014) Impact of SLM Build Parameters on the Surface Quality. RTejournal-Forum für Rapid Technologie, 11, 1-15.

[13] Nüsser, C. (2018) Laser Micro Polishing of Metals: Process Fundamentals. 3rd Conference on Laser Polishing, Aachen, 12-13 September 2018.

[14] Yadav, M.J., Jinoop, A.N., Danduk, C. and Subbu, S.K. (2017) Laser Shock Processing: Process Physics, Parameters, and Applications. Materials Today: Proceedings, 4, 7921-7930. https://doi.org/10.1016/j.matpr.2017.07.128

[15] Peyre, P., Fabbro, R., Berthe, L. and Dubouchet, C. (1996) Laser Shock Processing of Materials, Physical Processes Involved and Examples of Applications. Journal of Laser Applications, 8, 135-141. https://doi.org/10.2351/1.4745414

[16] Booij, S.M. (2003) Fluid Jet Polishing. Possibilities and Limitations of a New Fabrication Technique. PhD Dissertation, Technical University of Delft, Delft.

[17] Manfredi, D., Calignano, F., Krishnan, M., Canali, R., Ambrosio, E.P., Biamino, S., Ugues, D., Pavese, M. and Fino, P. (2014) Additive Manufacturing of Al Alloys and Aluminium Matrix Composites (AMCs). IntechOpen, London, 2.

https://doi.org/10.5772/58534

[18] Campanelli, S.L., Casalino, G., Contuzzi, N. and Ludovico, A.D. (2013) Taguchi Optimization of the Surface Finish Obtained by Laser Ablation on Selective Laser Molten Steel Parts. Procedia CIRP, 12, 462-467. https://doi.org/10.1016/j.procir.2013.09.079 
[19] Li, Y., Wu, Y., Zhou, L. and Fujimoto, M. (2014) Vibration-Assisted Dry Polishing of Fused Silica Using a Fixed-Abrasive Polisher. International Journal of Machine Tools and Manufacture, 77, 93-10. https://doi.org/10.1016/j.ijmachtools.2013.10.005

[20] Łyczkowska, E., Szymczyk, P., Dybała, B. and Chlebus, E. (2014) Chemical Polishing of Scaffolds Made of Ti-6Al-7Nb Alloy by Additive Manufacturing. Archives of Civil and Mechanical Engineering, 14, 586-594. https://doi.org/10.1016/j.acme.2014.03.001

[21] Bordatchev, E.V., Hafiz, A.M.K. and Tutunea-Fatan, O.R. (2014) Performance of Laser Polishing in Finishing of Metallic Surfaces. The International Journal of Advanced Manufacturing Technology, 73, 35-52. https://doi.org/10.1007/s00170-014-5761-3

[22] Ross, I. (2014) Prospects of Laser Polishing for Small and Complexly Shaped Parts. High Speed/High Precision Laser Microfabrication, Aachen, 14 June 2014. https://www.swissphotonics.net/libraries.files/epmt 2014 Ross.pdf

[23] Willenborg, E (2005) Polieren von Werkzeugstählen mit Laserstrahlung. PhD RWTH Aachen, Aachen.

[24] Burzic, B., Hofele, M., Mürdter, S. and Riegel, H. (2016) Laser Polishing of Ground Aluminum Surfaces with High Energy Continuous Wave Laser. Journal of Laser Applications, 29, Article ID: 011701. https://doi.org/10.2351/1.4966923

[25] Temmler, A., Liu, D., Luo, J. and Poprawe, R. (2020) Influence of Pulse Duration and Pulse Frequency on Micro-Roughness for Laser Micro Polishing $(\mathrm{L} \mu \mathrm{P})$ of Stainless Steel AISI 410. Applied Surface Science, 510, Article ID: 145272. https://doi.org/10.1016/j.apsusc.2020.145272

[26] Yasa, E., Deckers, J. and Kruth, J.-P. (2011) The Investigation of the Influence of Laser Remelting on Density, Surface Quality and Microstructure of Selective Laser Melting Parts. Rapid Prototyping Journal, 17, 312-327. https://doi.org/10.1108/13552541111156450

[27] Gora, W.S., et al. (2016) Enhancing Surface Finish of Additively Manufactured TiTanium and Cobalt Chrome Elements Using Laser Based Finishing. Physics Procedia, 83, 258-263. https://doi.org/10.1016/j.phpro.2016.08.021

[28] Yung, K.C., Xiao, T.Y., Choy, H.S., Wang, W.J. and Cai, Z.X. (2018) Laser Polishing of Additive Manufactured CoCr Alloy Components with Complex Surface Geometry. Journal of Materials Processing Technology, 262, 53-64. https://doi.org/10.1016/j.jmatprotec.2018.06.019

[29] Richter, B., Blanke, N., Werner, C., Vollertsen, F. and Pfefferkorn, F.E. (2019) Effect of Initial Surface Features on Laser Polishing of Co-Cr-Mo Alloy Made by Powder-Bed Fusion. JOM, 71, 912-919. https://doi.org/10.1007/s11837-018-3216-2

[30] Kumstel, J. (2015) Polieren von SLM-Bauteilen mit kontinuierlicher Laserstrahlung. In: Witt, G., Wegner, A. and Sehrt, J., Eds., Neue Entwicklungen in der Additiven Fertigung, Springer, Berlin, 143-157. https://doi.org/10.1007/978-3-662-48473-9 10

[31] Fang, Z., Lu, L., Chen, L. and Guan, Y. (2018) Laser Polishing of Additive Manufactured Superalloy. Procedia CIRP, 71, 150-154. https://doi.org/10.1016/j.procir.2018.05.088

[32] Dadbakhsh, S., Hao, L. and Kong, C.Y. (2010) Surface Finish Improvement of LMD Samples Using Laser Polishing. Virtual and Physical Prototyping, 5, 215-221. https://doi.org/10.1080/17452759.2010.528180

[33] Lamikiz, A., Sánchez, J.A., López de Lacalle, L.N. and Arana, J.L. (2007) Laser Polishing of Parts Built Up by Selective Laser Sintering. International Journal of Ma- 
chine Tools and Manufacture, 47, 2040-2050.

https://doi.org/10.1016/j.ijmachtools.2007.01.013

[34] Ukar, E., Lamikiz, A., Lacalle, L.L.D., Pozo, D.D., Liebana, F. and Sanchez, A. (2010) Laser Polishing Parameter Optimisation on Selective Laser Sintered Parts. International Journal of Machining and Machinability of Materials, 8, 417. https://doi.org/10.1504/IJMMM.2010.036148

[35] Breidenstein, B., Brenne, F., Wu, L., Niendorf, T. and Denkena, B. (2018) Effect of Post-Process Machining on Surface Properties of Additively Manufactured H13 Tool Steel. HTM Journal of Heat Treatment and Materials, 73, 173-186. https://doi.org/10.3139/105.110359

[36] Ma, C.P., Guan, Y.C. and Zhou, W. (2017) Laser Polishing of Additive Manufactured Ti Alloys. Optics and Lasers in Engineering, 93, 171-177.

https://doi.org/10.1016/j.optlaseng.2017.02.005

[37] Li, Y.-H., Wang, B., Ma, C.-P., Fang, Z.-H., Chen, L.-F., Guan, Y.-C. and Yang, S.-F. (2019) Material Characterization, Thermal Analysis, and Mechanical Performance of a Laser-Polished Ti Alloy Prepared by Selective Laser Melting. Metals, 9, 112. https://doi.org/10.3390/met9020112

[38] Schanz, J., et al. (2017) Metallurgical Investigations of Laser Remelted Additively Manufactured AlSi10Mg Parts. Materialwissenschaft und Werkstofftechnik, 48, 463 476. https://doi.org/10.1002/mawe.201700039

[39] Schanz, J., Hofele, M., Hitzler, L., Merkel, M. and Riegel, H. (2016) Laser Polishing of Additive Manufactured AlSi10Mg Parts with an Oscillating Laser Beam. In: Öchsner, A. and Altenbach, Eds., Machining, Joining and Modifications of Advanced Materials, Advanced Structured Materials, Springer, Singapore.

[40] Markovič, V., et al. (2016) Investigation of the Laser Polishing Influence on the Parts Built by SLS. Solid State Phenomena, 251, 8-13. https://doi.org/10.4028/www.scientific.net/SSP.251.8

[41] Hofele, M., Schanz, J., Roth, A., Harrison, D., Silva, A. and Riegel, H. (2020) Parameter Study of Continuous and Pulsed Wave Laser Polishing of Additive Manufactured Aluminum Parts. 